\title{
Unimodality of the Andrews-Garvan-Dyson cranks of partitions
}

\author{
Kathy Q. Ji ${ }^{1}$ and Wenston J.T. Zang ${ }^{2}$ \\ ${ }^{1}$ Center for Applied Mathematics, \\ Tianjin University, Tianjin 300072, P.R. China \\ ${ }^{2}$ Institute for Advanced Study in Mathematics, \\ Harbin Institute of Technology, Heilongjiang, 150001, P.R. China \\ ${ }^{1}$ kathyji@tju.edu.cn, $\quad 2$ zang@hit.edu.cn
}

\begin{abstract}
The main objective of this paper is to investigate the distribution of the Andrews-Garvan-Dyson cranks of partitions. Let $M(m, n)$ denote the number of partitions of $n$ with the Andrews-Garvan-Dyson crank $m$, we show that the sequence $\{M(m, n)\}_{|m| \leq n-1}$ is unimodal for $n \geq 44$. It turns out that the unimodality of $\{M(m, n)\}_{|m| \leq n-1}$ is related to the monotonicity properties of two partition functions $p_{r}(n)$ and $p p_{r}(n)$. Let $p_{r}(n)$ denote the number of partitions of $n$ with parts taken from $\{2,3, \ldots, r\}$ and let $p p_{r}(n)$ denote the number of pairs $(\alpha, \beta)$ of partitions, where $\alpha$ is a partition counted by $p_{r}(i)$ and $\beta$ is a partition counted by $p_{r+1}(n-i)$ for $0 \leq i \leq n$. We show that $p_{r}(n) \geq p_{r}(n-1)$ for $r \geq 5$ and $n \geq 14$ and $p p_{r}(n) \geq p p_{r}(n-1)$ for $r \geq 3$ and $n \geq 8$. With the aid of the monotonicity properties on $p_{r}(n)$ and $p p_{r}(n)$, we show that $M(m, n) \geq M(m, n-1)$ for $n \geq 14$ and $0 \leq m \leq n-2$ and $M(m-1, n) \geq M(m, n)$ for $n \geq 44$ and $1 \leq m \leq n-1$. By means of the symmetry $M(m, n)=M(-m, n)$, we find that $M(m-1, n) \geq M(m, n)$ for $n \geq 44$ and $1 \leq m \leq n-1$ implies that the sequence $\{M(m, n)\}_{|m| \leq n-1}$ is unimodal for $n \geq 44$. We also give a proof of an upper bound for ospt $(n)$ conjectured by Chan and Mao in light of $M(m-1, n) \geq M(m, n)$ for $n \geq 44$ and $0 \leq m \leq n-1$.
\end{abstract}

Keywords: Partition, rank, crank, unimodal, ospt-function

2010 Mathematics Subject Classification. 11P81, 05A17, 05A20

\section{Introduction}

Dyson's rank 21 and the Andrews-Garvan-Dyson crank [7] are two fundamental statistics in the theory of partitions. Recall that the rank of a partition was introduced by Dyson [21] as the largest part of the partition minus the number of parts. The crank of a partition was defined by Andrews and Garvan [7] as the largest part if the partition contains no ones, and otherwise as the number of parts larger than the number of ones minus the number of ones.

Let $p(n)$ denote the number of partitions of $n$. It was conjectured by Dyson [21] and confirmed by Atkin and Swinnerton-Dyer [11] that the rank of a partition could 
explain two of Ramanujan's famous partition congruences $p(5 n+4) \equiv 0(\bmod 5)$ and $p(7 n+5) \equiv 0(\bmod 7)$, but not the third one, $p(11 n+6) \equiv 0(\bmod 11)$. This led Dyson to hypothesize the existence of another statistic, namely the crank. Until forty-four years later, Andrews and Garvan [7], building on the work of Garvan [24] finally unveiled crank and showed that the crank can be used to interpret all three congruences on $p(n) \bmod$ 5, 7 and 11. For more details, please refer to Dyson [21, Atkin and Swinnerton-Dyer [11] and Andrews and Garvan [7,24]. It is worth mentioning that Mahlburg [30] showed that the crank can also provide combinatorial interpretations of infinite families of congruences on $p(n)$ established by Ahlgren and Ono [1] and Ono [31. Since then, the rank and the crank have been extensively studied, see, for example, Andrews and Garvan [8], Andrews and Ono [10, Bringmann and Dousse [12], Bringmann and Ono [15, 16], Garvan [26], Lewis [29], and so on.

Let $m$ be an integer. For $n \geq 1$, let $N(m, n)$ denote the number of partitions of $n$ with rank $m$, and for $n>1$, let $M(m, n)$ denote the number of partitions of $n$ with crank $m$. For $n=1$, set $M(0,1)=-1, M(1,1)=M(-1,1)=1$, and $M(m, 1)=0$ when $m \neq-1,0,1$. For $n=0$, set $M(0,0)=1$, and $M(m, 0)=0$ when $m \neq 0$. For $n<0$, set $M(m, n)=0$.

In 2014, Chan and Mao [17] showed the following two inequalities on $N(m, n)$ :

Theorem 1.1. (Chan and Mao). For $n \geq 12$ and $0 \leq m \leq n-3$ or $m=n-1$,

$$
N(m, n) \geq N(m, n-1) .
$$

Theorem 1.2. (Chan and Mao). For $m, n \geq 0$,

$$
N(m, n) \geq N(m+2, n) .
$$

In [5], Andrews, Chan and Kim introduced the function ospt( $n)$ defined as the difference between the first positive crank moment and the first positive rank moment, namely,

$$
\operatorname{ospt}(n)=\sum_{m=0}^{\infty} m M(m, n)-\sum_{m=0}^{\infty} m N(m, n)
$$

By means of generating function, Andrews, Chan and Kim [5] proved the positivity of $\operatorname{ospt}(n)$ and gave a combinatorial interpretation of $\operatorname{ospt}(n)$ which counts the number of even and odd strings in the partitions of $n$. Chen, Ji and Zang [19] gave another combinatorial interpretation of $\operatorname{ospt}(n)$ in terms of certain bijection.

Using Theorem 1.1 and Theorem 1.2, Chan and Mao [17] established the following upper-bound and lower-bound for ospt(n) in terms of $N(m, n), M(m, n)$ and $p(n)$.

Theorem 1.3. (Chan and Mao). The following inequalities are true.

$$
\operatorname{ospt}(n)>\frac{p(n)}{4}+\frac{N(0, n)}{2}-\frac{M(0, n)}{4}, \quad \text { for } n \geq 8,
$$




$$
\begin{array}{ll}
\operatorname{ospt}(n)<\frac{p(n)}{4}+\frac{N(0, n)}{2}-\frac{M(0, n)}{4}+\frac{N(1, n)}{2}, & \text { for } n \geq 7, \\
\operatorname{ospt}(n)<\frac{p(n)}{2}, & \text { for } n \geq 3 .
\end{array}
$$

At the end of the paper, Chan and Mao [17] raised a series of open problems, one of which is to establish similar inequalities for the crank of a partition. They also posed the following conjecture.

Conjecture 1.4. (Chan and Mao). For $n \geq 10$,

$$
\operatorname{ospt}(n)<\frac{p(n)}{3}
$$

In [28], Kim, Kim and Seo proved that $M(m, n)>M(m+1, n)$ for $m \geq 0$ and sufficiently large $n$. More precisely, they obtained the following result.

Theorem 1.5. (Kim, Kim and Seo). For $m \geq 0$,

$$
M(m, n)>M(m+1, n)
$$

for all positive integers $n>100$ satisfying

$$
\sqrt{n} I_{-9 / 2}\left(\pi \sqrt{\frac{2 n}{3}}\right)>217 \frac{(2 m+3)^{14}}{2 m+1} e^{\frac{\pi \sqrt{3}}{32}(2 m+3)^{2}} e^{\pi \sqrt{\frac{2 n}{3}}}
$$

where $I_{s}(z)$ is the modified Bessel function of the second kind.

In this paper, we establish the following two inequalities on $M(m, n)$.

Theorem 1.6. For $n \geq 14$ and $0 \leq m \leq n-2$,

$$
M(m, n) \geq M(m, n-1) .
$$

Theorem 1.7. For $n \geq 44$ and $1 \leq m \leq n-1$,

$$
M(m-1, n) \geq M(m, n) .
$$

Recall that a sequence $\left\{a_{i}\right\}_{1 \leq i \leq n}$ is unimodal if for some $1 \leq j \leq n$,

$$
a_{1} \leq \cdots \leq a_{j-1} \leq a_{j} \geq a_{j+1} \geq \cdots \geq a_{n}
$$

For more information, see [32, P.124, Ex.50].

From Theorem 1.7 and the symmetry $M(m, n)=M(-m, n)$ (see [23, 24]), we find the following unimodality of the crank. 


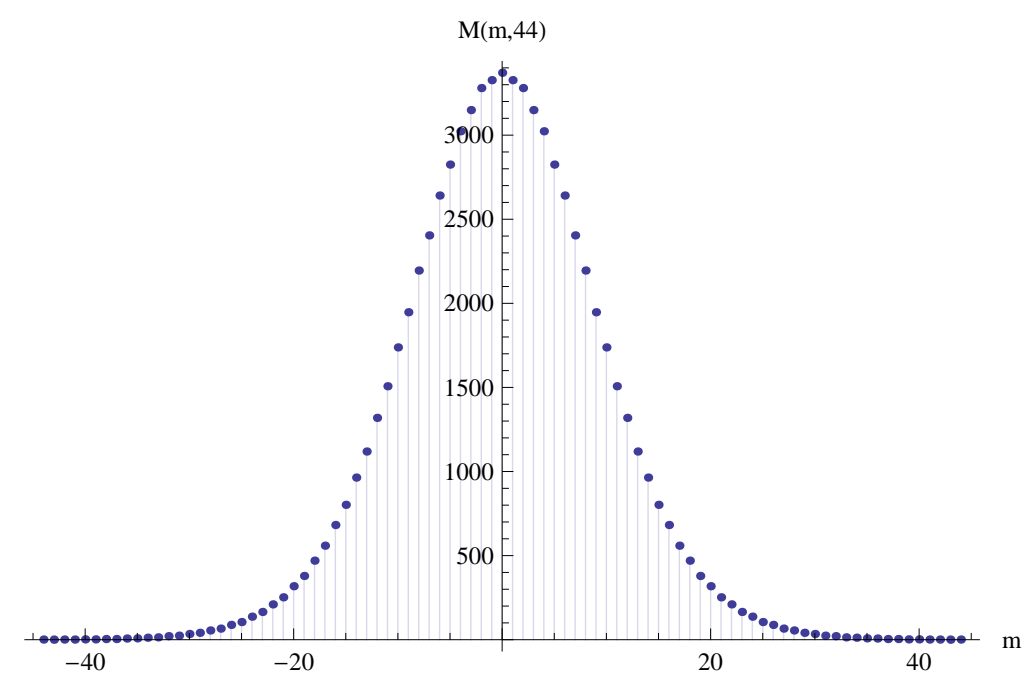

Figure 1.1: The sequence $\{M(m, 44)\}_{|m| \leq 43}$ is unimodal.

Corollary 1.8. For $n \geq 44$,

$$
M(1-n, n) \leq \cdots \leq M(-1, n) \leq M(0, n) \geq M(1, n) \geq \cdots \geq M(n-1, n) .
$$

That means the sequence $\{M(m, n)\}_{|m| \leq n-1}$ is unimodal for $n \geq 44$.

Fig. 1.1 gives an illustration of the unimodality of $\{M(m, 44)\}_{|m| \leq 43}$.

It should be noted that when

$$
(m, n) \in\{(2,5),(3,10),(4,9),(6,13)\}
$$

the inequality (1.8) does not hold.

Also, when

$$
\begin{aligned}
(m, n) \in & \{(1,2 i-1): 4 \leq i \leq 22\} \cup\{(2,4)\} \cup\{(2,2 i): 5 \leq i \leq 13\} \cup \\
& \{(3,5),(3,9),(3,15),(3,17),(3,21),(4,8),(4,10),(4,16),(5,9),(5,13),(6,12)\},
\end{aligned}
$$

the inequality (1.9) does not hold.

It is worth mentioning that Andrews, Dyson and Rhoades [6] conjectured the unimodality of the spt-crank defined on the spt-function. Let $N_{S}(m, n)$ denote the number of $S$-partitions of $n$ with spt-crank $m$, Andrews, Dyson and Rhoades conjectured that $\left\{N_{S}(m, n)\right\}_{m}$ is unimodal. Their conjecture was proved by Chen, Ji and Zang [18]. For the definitions of the spt-crank, the spt-function and the $S$-partition, please refer to [4] and [9].

In this paper, we also give a proof of Conjecture 1.4 in light of Theorem 1.7 and the following theorem. 
Theorem 1.9. For $n \geq 39$,

$$
p(n) \geq 21 M(0, n)
$$

The proofs of the two inequalities on $M(m, n)$ relates to the monotonicity property of the partition function $p_{r}(n)$, which counts the number of partitions of $n$ with parts taken from $\{2,3, \ldots, r\}$. From the definition of $p_{r}(n)$, it is easy to see that the generating function of $p_{r}(n)$ is

$$
\sum_{n=0}^{\infty} p_{r}(n) q^{n}=\frac{1}{\left(q^{2} ; q\right)_{r-1}}
$$

Here and throughout the rest of this paper, we adopt the common $q$-series notation [2]:

$$
(a ; q)_{\infty}=\prod_{n=0}^{\infty}\left(1-a q^{n}\right) \quad \text { and } \quad(a ; q)_{n}=\frac{(a ; q)_{\infty}}{\left(a q^{n} ; q\right)_{\infty}}
$$

We show that $p_{r}(n)$ has the following monotonicity property.

Theorem 1.10. For $r \geq 5$ and $n \geq 14$,

$$
p_{r}(n) \geq p_{r}(n-1) .
$$

It should be noted that Theorem 3.1 gives more results on $p_{r}(n)-p_{r}(n-1)$.

The proof of Theorem 1.7 for $m=2$ and $m=3$ also requires the monotonicity property of another partition function $p p_{r}(n)$. Let $p p_{r}(n)$ denote the number of pairs $(\alpha, \beta)$, where $\alpha$ is a partition counted by $p_{r}(i)$ and $\beta$ is a partition counted by $p_{r+1}(n-i)$ for $0 \leq i \leq n$. From the definition of $p p_{r}(n)$ and (1.11), it is easy to see that the generating function of $p p_{r}(n)$ is

$$
\sum_{n=0}^{\infty} p p_{r}(n) q^{n}=\frac{1}{\left(q^{2} ; q\right)_{r-1}\left(q^{2} ; q\right)_{r}} .
$$

We show that $p p_{r}(n)$ has the following monotonicity property.

Theorem 1.11. For $r \geq 3$ and $n \geq 8$,

$$
p p_{r}(n) \geq p p_{r}(n-1) \text {. }
$$

It should be noted that more results on $p p_{r}(n)-p p_{r}(n-1)$ are stated in Theorem 4.1.

This paper is organized as follows. In Section 2, we give a brief outline of the proofs of Theorem 1.6 and Theorem 1.7. In Section 3, we show the monotonicity property of $p_{r}(n)$. Section 4 is devoted to the proof of the monotonicity property of $p p_{r}(n)$. In Section 5, we give a proof of Theorem 1.6 by means of Theorem 3.1 and Corollary 3.3 . Sections $6 \sim 9$ are devoted to the proof of Theorem 1.7. More specifically, we establish three expressions for the generating function of $M(m-1, n)-M(m, n)$ in Section 6. Section 7 is devoted 
to the proof of Theorem 1.7 when $m=2$ in light of Theorem 4.1. In Section 8, we show Theorem 1.7 holds when $m \geq 3$ in light of Theorem 3.1, Corollary 3.3 and Theorem 4.1 , In Section 9, we finish the proof of Theorem 1.7 by showing that $M(0, n) \geq M(1, n)$ for $n \geq 44$. In Section 10, we first prove Theorem 1.9, and then confirm Conjecture 1.4 in light of Theorem 1.7 and Theorem 1.9. Finally, two conjectures on the log-concavity of $M(m, n)$ and $N(m, n)$ are posed in Section 11.

\section{The outline of the proofs of Theorems 1.6 and 1.7}

The proofs of Theorem 1.6 and Theorem 1.7 both rely on the generating function of $M(m, n)$ established by Garvan [25]:

Theorem 2.1. (Garvan). For $m \geq 0$,

$$
\sum_{n=0}^{\infty} M(m, n) q^{n}=\frac{(1-q) q^{m}}{(q ; q)_{m}}+\sum_{k=1}^{\infty} \frac{q^{k(k+m)+2 k+m}}{(q ; q)_{k}\left(q^{2} ; q\right)_{k+m-1}}
$$

By Theorem 2.1, it is easy to see that

$$
\begin{aligned}
& \sum_{n=0}^{\infty}(M(m, n)-M(m, n-1)) q^{n} \\
= & \frac{(1-q)^{2} q^{m}}{(q ; q)_{m}}+\frac{q^{2 m+3}}{\left(q^{2} ; q\right)_{m}}+\sum_{k=2}^{\infty} \frac{q^{k(k+m)+2 k+m}}{\left(q^{2} ; q\right)_{k-1}\left(q^{2} ; q\right)_{k+m-1}} .
\end{aligned}
$$

To prove Theorem 1.6, it suffices to show that the coefficients of $q^{n}$ in (2.2) are nonnegative when $n \geq 14$ and $0 \leq m \leq n-2$. By the definition (1.11) of $p_{r}(n)$ and (2.2), we see that for $m \geq 2$ and $n \geq m+1$,

$$
M(m, n)-M(m, n-1) \geq p_{m}(n-m)-p_{m}(n-m-1)+p_{m+1}(n-2 m-3) .
$$

With the aid of Theorem 3.1 and Corollary 3.3 , we will show that the right-hand side of (2.3) is nonnegative when $n \geq 14$ and $0 \leq m \leq n-2$, which leads to Theorem 1.6.

Similarly, by Theorem 2.1, we see that for $m \geq 1$,

$$
\sum_{n=0}^{\infty}(M(m-1, n)-M(m, n)) q^{n}
$$




$$
\begin{aligned}
= & \sum_{k=1}^{\infty} \frac{q^{k(k+m-1)+2 k+m-1}}{(q ; q)_{k}\left(q^{2} ; q\right)_{k+m-2}}-\sum_{k=1}^{\infty} \frac{q^{k(k+m)+2 k+m}}{(q ; q)_{k}\left(q^{2} ; q\right)_{k+m-1}} \\
& +\frac{(1-q) q^{m-1}}{(q ; q)_{m-1}}-\frac{q^{m}}{\left(q^{2} ; q\right)_{m-1}} .
\end{aligned}
$$

In order to prove Theorem [1.7, we aim to show that the coefficients of $q^{n}$ in (2.4) are nonnegative when $n \geq 44$ and $1 \leq m \leq n-1$. It turns out that this will be more difficult and it is required to transform (2.4) into several summations which have nonnegative power series coefficients. To this end, we first split the first summation in (2.4) into five summations as stated in Lemma 6.1, and then split the second summation in (2.4) into five summations as stated in Lemma 6.2. Based on Lemma 6.1 and Lemma 6.2, we could derive from (2.4) a new expression of the generating function of $M(m-1, n)-M(m, n)$ stated in Theorem 6.3. Moreover, when $m \geq 2$, it can be shown that some summations in Theorem 6.3 have nonnegative power series coefficients, see Theorem6.4 and Theorem6.5. The proof of Theorem 1.7 consists of three parts: (1) $m=2$ (Section 7), (2) $m \geq 3$ (Section 8), (3) $m=1$ (Section 9).

When $m=2$, based on Theorem 6.4, we will show that for $n \geq 15$,

$$
M(1, n)-M(2, n) \geq T_{2}(n)
$$

where $T_{2}(n)$ is defined as:

$$
\sum_{n=0}^{\infty} T_{2}(n) q^{n}:=\sum_{r=1}^{\infty} \frac{q^{r^{2}+7 r+7}(1-q)}{\left(q^{2} ; q\right)_{r}\left(q^{2} ; q\right)_{r+1}} .
$$

By the definition (1.13) of $p p_{r}(n)$, we see that

$$
\sum_{n=0}^{\infty} T_{2}(n) q^{n}=\sum_{r=1}^{\infty} q^{r^{2}+7 r+7} \sum_{n=0}^{\infty}\left(p p_{r+1}(n)-p p_{r+1}(n-1)\right) q^{n}
$$

In light of Theorem 4.1, we will show that $M(1, n)-M(2, n) \geq 0$ for $n \geq 44$.

When $m \geq 3$. In light of Theorem 6.5, we will show that for $n \geq 0$,

$$
M(m-1, n)-M(m, n) \geq U_{m}(n),
$$

where

$$
\sum_{n=0}^{\infty} U_{m}(n) q^{n}=-q^{2 m}+q^{2 m+1}+q^{3 m+1}+q^{m-1} \frac{1-q}{\left(q^{2} ; q\right)_{m-2}}+\frac{q^{4 m+8}}{\left(q^{2} ; q\right)_{m}}
$$


By the definition (1.11) of $p_{r}(n)$, we see that $U_{m}(n)$ can be expressed in terms of $p_{m}(n)$ and $p_{m}(n)-p_{m}(n-1)$. With the aid of Theorem 3.1 and Corollary 3.3, we will show that $U_{m}(n) \geq 0$ for $m \geq 3$ and $n \geq 44$, which implies $M(m-1, n) \geq M(m, n)$ for $m \geq 3$ and $n \geq 44$.

The proof of Theorem 1.7 when $m=1$ is the most complicated. It is required to do more operations on (2.4) when $m=1$. Besides Lemma 6.1 and Lemma 6.2, we also need three more lemmas ( Lemmas 9.2, 9.3, 9.4). Based on these five lemmas, we succeed to transform (2.4) when $m=1$ into several summations stated in Theorem 9.1 which have nonnegative power series coefficients. Using Theorem 9.1, we will deduce that $M(0, n)-M(1, n) \geq T_{1}(n)$ for $n \geq 10$, where $T_{1}(n)$ is defined as in (9.31). With the aid of a result of Chan and Mao [17, namely Lemma 3.5, and the exact formula of $p_{4}(n)$ stated in Lemma 3.2, we deduce that $T_{1}(n) \geq 0$ for $n \geq 106$. This leads to $M(0, n)-M(1, n) \geq 0$ for $n \geq 106$. Moreover, it can be checked that $M(0, n)-M(1, n) \geq 0$ when $44 \leq n \leq 105$. Therefore, we show that Theorem 1.7 holds when $m=1$.

\section{The monotonicity property of $p_{r}(n)$}

In this section, we aim to investigate the monotonicity property of $p_{r}(n)$. We will prove the following results on $p_{r}(n)-p_{r}(n-1)$ for $r \geq 2$, which leads to Theorem 1.10 immediately. The results for some special cases of $p_{r}(n)-p_{r}(n-1)$ will be used in the proofs of Theorem 1.6 and Theorem 1.7 .

Theorem 3.1. For $r \geq 2$, define

$$
d_{r}(n)=p_{r}(n)-p_{r}(n-1)
$$

Then

(1) $d_{r}(0)=1$ and $d_{r}(1)=-1$ for all $r \geq 2$.

(2) $d_{2}(n)=1$ when $n$ is even and $d_{2}(n)=-1$ when $n$ is odd.

(3) $d_{3}(n)=1$ when $n \equiv 0,2(\bmod 6), d_{3}(n)=-1$ when $n \equiv 1(\bmod 6)$ and $d_{3}(n)=0$ when $n \equiv 3,4,5(\bmod 6)$.

(4) $d_{4}(n)>0$ when $n$ is even, $d_{4}(n)=-\lfloor(n+11) / 12\rfloor$ when $n \equiv 1(\bmod 2)$ and $n \not \equiv 3$ $(\bmod 12)$ and $d_{4}(n)=-\lfloor n / 12\rfloor$ when $n \equiv 3(\bmod 12)$.

(5) $d_{5}(n) \geq 0$ for $n \geq 2$. Moreover, $d_{5}(n) \geq 1$ for $n \geq 14$.

(6) $d_{6}(n) \geq 0$ for $n \geq 0$ except for $d_{6}(1)=d_{6}(7)=d_{6}(13)=-1$.

(7) When $r \geq 7, d_{r}(n) \geq 0$ for $n \geq 2$. Moreover, $d_{r}(r+2) \geq 1$ and $d_{r}(2 r+7) \geq 1$. 
To prove Theorem 3.1, we first establish exact formulas for $p_{r}(n)$ when $2 \leq r \leq 4$ in Lemma 3.2. We then establish three expressions for the generating function of $p_{r}(n)$ in Lemma 3.4. We proceed to show Lemma 3.6, which plays a crucial role in the proof of Theorem [3.1. Finally, we give a proof of Theorem 3.1 based on Lemma 3.2, Lemma 3.4 and Lemma 3.6.

Lemma 3.2. When $2 \leq r \leq 4$, we have the following explicit formulas for $p_{r}(n)$ :

$$
p_{2}(n)= \begin{cases}1, & \text { if } n \text { is even } \\ 0, & \text { if } n \text { is odd }\end{cases}
$$

(2)

$$
p_{3}(n)=\left\{\begin{array}{lll}
\left\lfloor\frac{n}{6}\right\rfloor+1, & \text { if } n \neq 1 \quad(\bmod 6) \\
\left\lfloor\frac{n}{6}\right\rfloor, & \text { if } n \equiv 1 \quad(\bmod 6)
\end{array}\right.
$$

(3)

$$
p_{4}(n)= \begin{cases}3 a^{2}+3 a+1, & \text { if } n=12 a \text { or } n=12 a+3 ; \\ 3 a^{2}+4 a+1, & \text { if } n=12 a+2 \text { or } n=12 a+5 ; \\ 3 a^{2}+5 a+2, & \text { if } n=12 a+4 \text { or } n=12 a+7 ; \\ 3 a^{2}+6 a+3, & \text { if } n=12 a+6 \text { or } n=12 a+9 ; \\ 3 a^{2}+7 a+4, & \text { if } n=12 a+8 \text { or } n=12 a+11 ; \\ 3 a^{2}+8 a+5, & \text { if } n=12 a+10 \text { or } n=12 a+13 .\end{cases}
$$

Proof. Let $p(n, r)$ denote the number of partitions of $n$ with at most $r$ parts. It is well known that

$$
\sum_{n=0}^{\infty} p(n, r) q^{n}=\frac{1}{(q ; q)_{r}}
$$

From (1.11) and (3.5), we see that

$$
\sum_{n=0}^{\infty} p_{r}(n) q^{n}=\frac{1-q}{(q ; q)_{r}}=\sum_{n=0}^{\infty} p(n, r) q^{n}-\sum_{n=1}^{\infty} p(n-1, r) q^{n} .
$$

Therefore, we find that for $n \geq 1$,

$$
p_{r}(n)=p(n, r)-p(n-1, r) .
$$

When $r=2$, Andrews [3] showed that

$$
p(n, 2)=\left\lfloor\frac{n+2}{2}\right\rfloor .
$$


DeMorgan [20] found the following formula for $p(n, 3)$ as given below,

$$
p(n, 3)=\left\{\frac{(n+3)^{2}}{12}\right\}
$$

Glösel [27] gave the following formula for $p(n, 4)$,

$$
p(n, 4)=\left\{\left\lfloor\frac{(n+4)}{2}\right\rfloor^{2}\left(3\left\lfloor\frac{n+9}{2}\right\rfloor-\left\lfloor\frac{n+10}{2}\right\rfloor\right) \frac{1}{36}\right\},
$$

where $\lfloor x\rfloor$ is the greatest integer $\leq x$, and $\{x\}$ is the nearest integer to $x$. Substituting (3.8), (3.9) and (3.10) into (3.7), and after some calculations, we see that (3.2), (3.3) and (3.4) hold.

By Lemma [3.2, we obtain the following corollary, which is useful in the proof of Theorem [1.6.

Corollary 3.3. For $r \geq 3$ and $n \geq 2$, we have $p_{r}(n) \geq 1$. Moreover, $p_{r}(n) \geq\left\lfloor\frac{n}{6}\right\rfloor$.

Proof. By the definition of $p_{r}(n)$, it is clear to see that for any $i \geq 2$, each partition counted by $p_{i}(n)$ is also counted by $p_{i+1}(n)$. So

$$
p_{r}(n) \geq p_{r-1}(n) \geq \cdots \geq p_{3}(n) .
$$

Furthermore, it is clear from Lemma 3.2 that $p_{3}(n) \geq 1$ for $n \geq 2$. Moreover, $p_{3}(n) \geq\left\lfloor\frac{n}{6}\right\rfloor$. This yields the corollary.

The following lemma gives three expressions for the generating function of $p_{r}(n)$. To be specific, the expression (3.12) will be used in the proof of Theorem 6.5 and the expressions (3.13) and (3.14) will be used in the proof of Theorem 3.1 .

Lemma 3.4. For $r \geq 2$. we have

$$
\begin{aligned}
\sum_{n=0}^{\infty} p_{r}(n) q^{n} & =1+\sum_{j=2}^{r} \frac{q^{j}}{\left(q^{j} ; q\right)_{r-j+1}} \\
& =1-q+\frac{q}{\left(q^{2} ; q\right)_{r-2}}+\sum_{j=1}^{r} \frac{q^{2 j}}{\left(q^{2} ; q\right)_{j-1}} \\
& =q^{r}+\frac{1}{\left(q^{2} ; q\right)_{r-2}}+\frac{q^{2 r}}{\left(q^{2} ; q\right)_{r-1}}+\sum_{j=2}^{r-1} \frac{q^{r+j}}{\left(q^{2} ; q\right)_{j-1}}
\end{aligned}
$$


Proof. We first verify (3.12). For $2 \leq j \leq r$, let $p_{r, j}(n)$ denote the number of partitions of $n$ such that each part is not exceeding $r$ and the smallest part is equal to $j$. Clearly, for $n \geq 1$,

$$
\sum_{j=2}^{r} p_{r, j}(n)=p_{r}(n)
$$

On the other hand, it is easy to see that

$$
\sum_{n=1}^{\infty} p_{r, j}(n) q^{n}=\frac{q^{j}}{\left(q^{j} ; q\right)_{r-j+1}}
$$

Combining (3.15) and (3.16), we obtain

$$
\sum_{n=0}^{\infty} p_{r}(n) q^{n}=1+\sum_{j=2}^{r} \sum_{n=1}^{\infty} p_{r, j}(n) q^{n}=1+\sum_{j=2}^{r} \frac{q^{j}}{\left(q^{j} ; q\right)_{r-j+1}}
$$

which is (3.12).

We proceed to derive (3.13). To this end, we need to divide the set of partitions counted by $p_{r}(n)$ into two disjoint subsets based on the difference of the largest part of the partition and the second largest part. Let $s_{r}(n)$ denote the number of partitions $\lambda=\left(\lambda_{1}, \lambda_{2}, \ldots, \lambda_{\ell}\right)$ counted by $p_{r}(n)$ such that $\lambda_{1}-\lambda_{2} \geq 1$ and $q_{r}(n)$ denote the number of partitions $\lambda=\left(\lambda_{1}, \lambda_{2}, \ldots, \lambda_{\ell}\right)$ counted by $p_{r}(n)$ such that $\lambda_{1}-\lambda_{2}=0$. Here we use the convention that $\lambda_{i}=0$ for $i>\ell$. Obviously,

$$
p_{r}(n)=s_{r}(n)+q_{r}(n) .
$$

Hence the generating function of $p_{r}(n)$ is equal to the sum of the generating functions of $s_{r}(n)$ and $q_{r}(n)$.

We first consider the generating function of $s_{r}(n)$. Let $\lambda=\left(\lambda_{1}, \lambda_{2}, \ldots, \lambda_{\ell}\right)$ be a partition counted by $s_{r}(n)$. If $\lambda \neq(2)$ note that $\lambda_{1}>\lambda_{2}$. Then we can define $\mu=$ $\left(\lambda_{1}-1, \lambda_{2}, \ldots, \lambda_{\ell}\right)$ which clearly is a partition counted by $p_{r-1}(n-1)$. Hence, by (1.11), we obtain the following generating function of $s_{r}(n)$ :

$$
\sum_{n=0}^{\infty} s_{r}(n) q^{n}=-q+q^{2}+\frac{q}{\left(q^{2} ; q\right)_{r-2}}
$$

To establish the generating function of $q_{r}(n)$, we will classify the set of partitions counted by $q_{r}(n)$ based on the size of the largest part. Given $2 \leq j \leq r$, let $q_{r, j}(n)$ denote the number of partitions counted by $q_{r}(n)$ with the largest part $j$. By definition, we see that the generating function of $q_{r, j}(n)$ is equal to

$$
\sum_{n=0}^{\infty} q_{r, j}(n) q^{n}=\frac{1}{1-q^{2}} \frac{1}{1-q^{3}} \cdots \frac{1}{1-q^{j-1}} \frac{q^{2 j}}{1-q^{j}}=\frac{q^{2 j}}{\left(q^{2} ; q\right)_{j-1}} .
$$


Notice that the empty partition of 0 is counted by $q_{r}(n)$, so

$$
\sum_{n=0}^{\infty} q_{r}(n) q^{n}=1+\sum_{j=2}^{r} \sum_{n=0}^{\infty} q_{r, j}(n) q^{n}=1+\sum_{j=2}^{r} \frac{q^{2 j}}{\left(q^{2} ; q\right)_{j-1}} .
$$

Combining (3.17) and (3.19), we obtain (3.13).

We finish the proof of Lemma 3.2 by showing (3.14) holds. We first divide the set of partitions counted by $p_{r}(n)$ into three disjoint sets. Let $g_{r}(n)$ denote the number of partitions $\lambda=\left(\lambda_{1}, \lambda_{2}, \ldots, \lambda_{\ell}\right)$ counted by $p_{r}(n)$ with $r=\lambda_{1}=\lambda_{2}$ and $h_{r}(n)$ denote the number of partitions $\lambda=\left(\lambda_{1}, \lambda_{2}, \ldots, \lambda_{\ell}\right)$ counted by $p_{r}(n)$ with $r=\lambda_{1}>\lambda_{2}$. Note that the number of partitions $\lambda=\left(\lambda_{1}, \lambda_{2}, \ldots, \lambda_{\ell}\right)$ counted by $p_{r}(n)$ with $r>\lambda_{1}$ is equal to $p_{r-1}(n)$, hence

$$
p_{r}(n)=p_{r-1}(n)+g_{r}(n)+h_{r}(n) .
$$

By (1.11), we see that the generating function of $p_{r-1}(n)$ is

$$
\sum_{n=0}^{\infty} p_{r-1}(n) q^{n}=\frac{1}{\left(q^{2} ; q\right)_{r-2}}
$$

Notice that $g_{r}(n)$ coincides with $q_{r, r}(n)$. Hence by (3.18), we see that

$$
\sum_{n=0}^{\infty} g_{r}(n) q^{n}=\frac{q^{2 r}}{\left(q^{2} ; q\right)_{r-1}}
$$

To obtain the generating function of $h_{r}(n)$, we define $h_{r, j}(n)$ as the number of partitions $\lambda=\left(\lambda_{1}, \lambda_{2}, \ldots, \lambda_{\ell}\right)$ counted by $h_{r}(n)$ with the second largest part $\lambda_{2}=j$. If $j=0$, then $\lambda=(r)$. Otherwise, for $2 \leq j \leq r-1$, the generating function of $h_{r, j}(n)$ is equal to

$$
\sum_{n=0}^{\infty} h_{r, j}(n) q^{n}=\frac{1}{1-q^{2}} \frac{1}{1-q^{3}} \cdots \frac{1}{1-q^{j-1}} \frac{q^{j}}{1-q^{j}} q^{r}=\frac{q^{r+j}}{\left(q^{2} ; q\right)_{j-1}}
$$

Hence, we obtain the following generating function of $h_{r}(n)$

$$
\sum_{n=0}^{\infty} h_{r}(n) q^{n}=q^{r}+\sum_{j=2}^{r-1} \sum_{n=0}^{\infty} h_{r, j}(n) q^{n}=q^{r}+\sum_{j=2}^{r-1} \frac{q^{r+j}}{\left(q^{2} ; q\right)_{j-1}} .
$$

Combining (3.20), (3.21) and (3.23), we obtain (3.14). This completes the proof.

Before proceeding to prove the monotonicity property of $p_{r}(n)$, let us first show Lemma 3.6, which plays a crucial role in the proof of Theorem 3.1. It turns out that the following lemma due to Chan and Mao [17] is needed in the proof of Lemma 3.6. 
Lemma 3.5. (Chan and Mao).

$$
\frac{1-q^{m}}{\left(1-q^{2}\right)\left(1-q^{3}\right)}
$$

has nonnegative power series coefficients for any integer $m \geq 2$.

Lemma 3.6. For $r \geq 4$, let

$$
\sum_{n=0}^{\infty} t_{r}(n) q^{n}:=\sum_{j=2}^{r} \frac{q^{2 j}\left(1-q^{r-j+2}\right)}{\left(q^{2} ; q\right)_{j-1}} .
$$

Then $t_{r}(n) \geq 0$ for $n \geq 0$. Moreover, when $r \neq 5$, we have $t_{r}(n) \geq 1$ for $n \geq 14$.

Proof. Define

$$
\sum_{n=0}^{\infty} t_{r}^{(j)}(n) q^{n}=\frac{q^{2 j}\left(1-q^{r-j+2}\right)}{\left(q^{2} ; q\right)_{j-1}}
$$

obviously,

$$
t_{r}(n)=\sum_{j=2}^{r} t_{r}^{(j)}(n) .
$$

By Lemma 3.5. we see that when $r \geq 4$ and $3 \leq j \leq r$,

$$
\sum_{n=0}^{\infty} t_{r}^{(j)}(n) q^{n}=\frac{q^{2 j}\left(1-q^{r-j+2}\right)}{\left(q^{2} ; q\right)_{j-1}}=\frac{1-q^{r-j+2}}{\left(1-q^{2}\right)\left(1-q^{3}\right)} \cdot \frac{q^{2 j}}{\left(q^{4} ; q\right)_{j-3}}
$$

has nonnegative power series coefficients. It gives that when $r \geq 4$ and $3 \leq j \leq r$,

$$
t_{r}^{(j)}(n) \geq 0 \text { for } n \geq 0 .
$$

We next show that $t_{r}^{(2)}(n)+t_{r}^{(3)}(n) \geq 0$ when $r \neq 5$. First, it is easy to see that

$$
\begin{aligned}
\sum_{n=0}^{\infty} t_{r}^{(2)}(n) q^{n}+\sum_{n=0}^{\infty} t_{r}^{(3)}(n) q^{n} & =\frac{q^{4}\left(1-q^{r}\right)}{1-q^{2}}+\frac{q^{6}\left(1-q^{r-1}\right)}{\left(1-q^{2}\right)\left(1-q^{3}\right)} \\
& =\frac{q^{4}-q^{7}+q^{6}-q^{r+2}+q^{r+2}-q^{r+4}-q^{r+5}+q^{r+7}}{\left(1-q^{2}\right)\left(1-q^{3}\right)} \\
& =\frac{q^{4}}{1-q^{2}}+\frac{q^{6}-q^{r+2}}{\left(1-q^{2}\right)\left(1-q^{3}\right)}+q^{r+2}
\end{aligned}
$$

By using Lemma 3.5 again, we find that when $r \geq 4$ and $r \neq 5$,

$$
\frac{q^{6}-q^{r+2}}{\left(1-q^{2}\right)\left(1-q^{3}\right)}=\frac{q^{6}\left(1-q^{r-4}\right)}{\left(1-q^{2}\right)\left(1-q^{3}\right)}
$$


has nonnegative power series coefficients. Hence, from (3.27), we see that when $r \neq 5$,

$$
t_{r}^{(2)}(n)+t_{r}^{(3)}(n) \geq 0 \quad \text { for } \quad n \geq 0 .
$$

Thus, we derive from (3.25) and (3.26) that $t_{r}(n) \geq 0$ when $r \neq 5$.

We next show that when $r \neq 5, t_{r}(n) \geq 1$ for $n \geq 14$. By the generating function (1.11) of $p_{r}(n)$, we see that

$$
\sum_{n=0}^{\infty} t_{r}^{(4)}(n) q^{n}=\frac{q^{8}\left(1-q^{r-2}\right)}{\left(1-q^{2}\right)\left(1-q^{3}\right)\left(1-q^{4}\right)}=\sum_{n=8}^{\infty}\left(p_{4}(n-8)-p_{4}(n-r-6)\right) q^{n} .
$$

From Lemma 3.2 (3), it is easy to check that for $n \geq 14$,

$$
p_{4}(n-8)>p_{4}(n-10) \text {, }
$$

and

$$
p_{4}(n-8)>p_{4}(n-13)
$$

Hence when $r \geq 4$ even,

$$
p_{4}(n-8)>p_{4}(n-10) \geq \cdots \geq p_{4}(n-r-6),
$$

and when $r \geq 7$ odd,

$$
p_{4}(n-8)>p_{4}(n-13) \geq p_{4}(n-15) \geq \cdots \geq p_{4}(n-r-6) .
$$

In either case, we see that $p_{4}(n-8)>p_{4}(n-r-6)$ for $n \geq 14$. It yields that when $r \neq 5$,

$$
t_{r}^{(4)}(n) \geq 1 \text { for } n \geq 14 \text {. }
$$

Combining (3.26), (3.28) and (3.30), it follows from (3.25) that when $r \neq 5, t_{r}(n) \geq 1$ for $n \geq 14$. Thus, we complete the proof of Lemma 3.6 when $r \neq 5$.

It remains to show that Lemma 3.6 holds when $r=5$. From (3.26), we see that for $3 \leq j \leq 5$,

$$
t_{5}^{(j)}(n) \geq 0
$$

Note that

$$
\sum_{n=0}^{\infty} t_{5}^{(2)}(n) q^{n}=\frac{q^{4}\left(1-q^{5}\right)}{\left(1-q^{2}\right)}=\frac{q^{4}}{1-q^{2}}-\frac{q^{9}}{1-q^{2}}=\sum_{n=2}^{\infty} q^{2 n}-\sum_{n=4}^{\infty} q^{2 n+1},
$$

so we derive that $t_{5}^{(2)}(n)=1$ when $n$ is even and $n \geq 4$ and $t_{5}^{(2)}(n)=-1$ when $n$ is odd and $n \geq 9$. Since

$$
\sum_{n=0}^{\infty} t_{5}^{(5)}(n) q^{n}=\frac{q^{10}}{\left(q^{3} ; q\right)_{3}}=\sum_{i=0}^{\infty} \sum_{j=0}^{\infty} \sum_{h=0}^{\infty} q^{3 i+4 j+5 h+10},
$$

and it is easy to check that for $n \geq 13$, there exists nonnegative integers $i, j, h$ such that $3 i+4 j+5 h+10=n$. Hence $t_{5}^{(5)}(n) \geq 1$. Thus, from the above analysis, we derive that

$$
t_{5}(n) \geq t_{5}^{(2)}(n)+t_{5}^{(5)}(n) \geq 0 \text { for } n \geq 13 .
$$

It is trivial to check that $t_{5}(n) \geq 0$ for $0 \leq n \leq 12$. Hence Lemma 3.6 is also valid when $r=5$. Thus, we complete the proof of the lemma. 
We are now in a position to give a proof of Theorem 3.1 .

Proof of Theorem 3.1. From (3.1) and Lemma 3.2, it is easy to check that Theorem 3.1 holds when $r=2,3$, or 4 .

We now consider the case $r \geq 5$. By (3.13), we see that

$$
\sum_{n=0}^{\infty} p_{r}(n) q^{n}=1-q+\frac{q}{\left(q^{2} ; q\right)_{r-2}}+\sum_{j=1}^{r} \frac{q^{2 j}}{\left(q^{2} ; q\right)_{j-1}},
$$

and from (3.14),

$$
\sum_{n=1}^{\infty} p_{r}(n-1) q^{n}=q^{r+1}+\frac{q}{\left(q^{2} ; q\right)_{r-2}}+\frac{q^{2 r+1}}{\left(q^{2} ; q\right)_{r-1}}+\sum_{j=2}^{r-1} \frac{q^{r+j+1}}{\left(q^{2} ; q\right)_{j-1}} .
$$

Hence, we have the following generating function of $d_{r}(n)$ :

$$
\sum_{n=0}^{\infty} d_{r}(n) q^{n}=1-q-q^{r+1}-\frac{q^{2 r+1}}{\left(q^{2} ; q\right)_{r-1}}+\sum_{j=1}^{r} \frac{q^{2 j}}{\left(q^{2} ; q\right)_{j-1}}-\sum_{j=2}^{r-1} \frac{q^{r+j+1}}{\left(q^{2} ; q\right)_{j-1}},
$$

which can be simplified as

$$
\sum_{n=0}^{\infty} d_{r}(n) q^{n}=1-q+q^{2}-q^{r+1}+q^{2 r} \frac{1-q}{\left(q^{2} ; q\right)_{r-1}}+\sum_{j=2}^{r-1} \frac{q^{2 j}\left(1-q^{r-j+1}\right)}{\left(q^{2} ; q\right)_{j-1}} .
$$

From (1.11) and (3.1), it is easy to see that

$$
q^{2 r} \frac{1-q}{\left(q^{2} ; q\right)_{r-1}}=\sum_{n=2 r}^{\infty} d_{r}(n-2 r) q^{n} .
$$

Moreover, using the notation of $t_{r}(n)$ as defined in (3.24), we see that (3.32) can be expressed as

$$
\sum_{n=0}^{\infty} d_{r}(n) q^{n}=1-q+q^{2}-q^{r+1}+\sum_{n=2}^{\infty} t_{r-1}(n) q^{n}+\sum_{n=2 r}^{\infty} d_{r}(n-2 r) q^{n} .
$$

Hence we obtain the following recurrence relation:

$$
d_{r}(n)= \begin{cases}t_{r-1}(n)+1, & \text { if } n=2 \text { or } 2 r \\ t_{r-1}(n)-1, & \text { if } n=r+1 \text { or } 2 r+1 \\ t_{r-1}(n), & \text { if } 3 \leq n \leq 2 r-1 \text { and } n \neq r+1 \\ t_{r-1}(n)+d_{r}(n-2 r), & \text { if } n \geq 2 r+2 .\end{cases}
$$


We next show that Theorem 3.1 holds when $r \geq 5$. From Lemma 3.6, we see that $t_{r-1}(n) \geq 0$ for $r \geq 5$ and $n \geq 2$. Moreover, when $r=5$ or $r \geq 7, t_{r-1}(n) \geq 1$ for $n \geq 14$, which implies that $t_{r-1}(r+1) \geq 1$ and $t_{r-1}(2 r+1) \geq 1$ when $r \geq 13$. By a simple calculation, we find that $t_{r-1}(r+1) \geq 1$ for $5 \leq r \leq 12$ and $r \neq 6$; and $t_{4}(11) \geq 1$. It follows that $t_{r-1}(r+1) \geq 1$ and $t_{r-1}(2 r+1) \geq 1$ when $r=5$ or $r \geq 7$. Hence, by (3.34), we derive that when $r=5$ or $r \geq 7$,

$$
d_{r}(n) \geq 0 \quad \text { for } 2 \leq n \leq 2 r+1
$$

and when $r=6$,

$$
d_{6}(n) \geq 0 \quad \text { for } 2 \leq n \leq 12 \text { and } n \neq 7 .
$$

We proceed to show that when $r \geq 5, d_{r}(n) \geq 0$ for $n \geq 2 r+2$ by using induction on $n$. Assume that there exists a positive integer $N_{r} \geq 2 r+1$ such that when $r=5$ or $r \geq 7$, $d_{r}(n) \geq 0$ for $2 \leq n \leq N_{r}$ and $d_{6}(n) \geq 0$ for $2 \leq n \leq N_{6}$ and $n \neq 7,13$.

We proceed to show that $d_{r}\left(N_{r}+1\right) \geq 0$ when $r \geq 5$. By (3.34) and the fact that $N_{r}+1 \geq 2 r+2$, we have

$$
d_{r}\left(N_{r}+1\right)=t_{r-1}\left(N_{r}+1\right)+d_{r}\left(N_{r}-2 r+1\right)
$$

From Lemma 3.6. we see that $t_{r-1}\left(N_{r}+1\right) \geq 0$ when $r \geq 5$ and by the induction hypothesis, we see that when $r=5$ or $r \geq 7, d_{r}\left(N_{r}+1-2 r\right) \geq 0$ and $d_{6}\left(N_{6}-11\right) \geq 0$ for $N_{6} \neq 18$ or 24 . Hence we derive from (3.37) that when $r=5$ or $r \geq 7, d_{r}\left(N_{r}+1\right) \geq 0$ and $d_{6}\left(N_{6}+1\right) \geq 0$ for $N_{6} \neq 18$ or 24 . Moreover, it is easy to check that $d_{6}(19) \geq 0$ and $d_{6}(25) \geq 0$. Thus, we conclude that when $r=5$ or $r \geq 7, d_{r}(n) \geq 0$ for $n \geq 2$ and $d_{6}(n) \geq 0$ for $n \geq 14$.

We finish the proof of Theorem 3.1 by considering the positivity of $d_{r}(n)$ when $r=5$ or $r \geq 7$. When $r=5$, and by (3.34), we see that when $n \geq 14$,

$$
d_{5}(n)=t_{4}(n)+d_{5}(n-10) .
$$

Since $t_{4}(n) \geq 1$ for $n \geq 14$ and $d_{5}(n-10) \geq 0$ for $n \geq 14$, we deduce that $d_{5}(n) \geq 1$ for $n \geq 14$. Thus we complete the proof of Theorem 3.1 when $r=5$.

From Lemma 3.6, we see that $t_{r-1}(n) \geq 1$ for $n \geq 14$ and $r \geq 7$. It follows from (3.34) that $d_{r}(r+2)=t_{r-1}(r+2) \geq 1$ when $r \geq 12$. Furthermore, it is easy to check that $d_{r}(r+2) \geq 1$ when $7 \leq r \leq 11$. So $d_{r}(r+2) \geq 1$ when $r \geq 7$. On the other hand, by (3.34), we see that

$$
d_{r}(2 r+7)=t_{r-1}(2 r+7)+d_{r}(7) .
$$

Note that $t_{r-1}(2 r+7) \geq 1$ when $r \geq 7$ and $d_{r}(7) \geq 0$ when $r \geq 7$, so we arrive at $d_{r}(2 r+7) \geq 1$. Thus we complete the proof of Theorem 3.1 . 


\section{The monotonicity property of $p p_{r}(n)$}

This section is devoted to the monotonicity property of $p p_{r}(n)$ in terms of Theorem 3.1 . We will show the following results on $p p_{r}(n)-p p_{r}(n-1)$, which gives Theorem 1.11 immediately. The results for some special cases of $p p_{r}(n)-p p_{r}(n-1)$ will be used in the proof of Theorem 1.7 when $m=2$.

Theorem 4.1. For $r \geq 2$, let

$$
f_{r}(n)=p p_{r}(n)-p p_{r}(n-1)
$$

Then

(1) $f_{r}(0)=1$ and $f_{r}(1)=-1$.

(2) $f_{2}(n) \geq 0$ if and only if $n$ is even. Moreover, $f_{2}(n)=-\left\lceil\frac{n}{6}\right\rceil$ when $n$ is odd.

(3) $f_{3}(n) \geq 0$ for $n \geq 2$ and $n \neq 7$. Moreover, $f_{3}(3)=f_{3}(5)=0, f_{3}(7)=-1$ and $f_{3}(n) \geq(n-15) / 2$ when $n$ is odd and $n \geq 17$.

(4) When $r \geq 4, f_{r}(n) \geq 0$ for $n \geq 2$. Moreover, $f_{r}(2 r+7) \geq 1$.

Proof. (1) From the definition of $f_{r}(n)$, and by (1.13), we see that

$$
\sum_{n=0}^{\infty} f_{r}(n) q^{n}=\frac{1-q}{\left(q^{2} ; q\right)_{r-1}\left(q^{2} ; q\right)_{r}}
$$

Clearly $f_{r}(0)=1$ and $f_{r}(1)=-1$.

(2) When $r=2$, we see that

$$
\sum_{n=0}^{\infty} f_{2}(n) q^{n}=\frac{1-q}{\left(1-q^{2}\right)\left(q^{2} ; q\right)_{2}}=\frac{1}{1-q^{2}} \sum_{n=0}^{\infty} d_{3}(n) q^{n} .
$$

From Theorem 3.1 (2), we find that

$$
\sum_{n=0}^{\infty} f_{2}(n) q^{n}=\frac{1}{1-q^{2}} \sum_{i=0}^{\infty}\left(q^{6 i}-q^{6 i+1}+q^{6 i+2}\right)
$$

which implies that

$$
\sum_{n=0}^{\infty} f_{2}(2 n) q^{2 n}=\frac{1}{1-q^{2}} \sum_{i=0}^{\infty}\left(q^{6 i}+q^{6 i+2}\right)
$$

and

$$
\sum_{n=0}^{\infty} f_{2}(2 n+1) q^{2 n+1}=-\frac{1}{1-q^{2}} \sum_{i=0}^{\infty} q^{6 i+1}=-\sum_{i=0}^{\infty} \sum_{j=0}^{\infty} q^{6 i+2 j+1}
$$


Hence $f_{2}(2 n) \geq 0$ and $f_{2}(2 n+1)=-\left\lceil\frac{2 n+1}{6}\right\rceil$ for $n \geq 0$. Thus we complete the proof of Theorem 4.1 when $r=2$.

(3) When $r=3$, we see that

$$
\begin{aligned}
\sum_{n=0}^{\infty} f_{3}(n) q^{n} & =\frac{1-q}{\left(q^{2} ; q\right)_{2}\left(q^{2} ; q\right)_{3}} \\
& =\frac{1-q}{\left(q^{2} ; q\right)_{4}} \cdot \frac{1-q^{5}}{\left(1-q^{2}\right)\left(1-q^{3}\right)} \\
& =\left(\sum_{n=0}^{\infty} d_{5}(n) q^{n}\right)\left(\frac{1}{1-q^{3}}+\frac{q^{2}}{1-q^{2}}\right)
\end{aligned}
$$

Define

$$
\sum_{n=0}^{\infty} s(n) q^{n}=\left(1-q+q^{4}+\sum_{\substack{n \geq 15 \\ n \text { odd }}} q^{n}\right)\left(\frac{1}{1-q^{3}}+\frac{q^{2}}{1-q^{2}}\right)
$$

From Theorem 3.1 (5), we see that $d_{5}(n) \geq 1$ for $n \geq 14$ and note that $d_{5}(4) \geq 1$. Hence, we deduce that for $n \geq 0$,

$$
f_{3}(n) \geq s(n)
$$

Observe that

$$
\sum_{n=0}^{\infty} s(n) q^{n}=\left(1-q+q^{4}+q^{15}\right)\left(\frac{1}{1-q^{3}}+\frac{q^{2}}{1-q^{2}}\right)+\sum_{\substack{n \geq 17 \\ n \text { odd }}} q^{n}\left(\frac{1}{1-q^{3}}+\frac{q^{2}}{1-q^{2}}\right)
$$

and note that

$$
\begin{aligned}
& \left(1-q+q^{4}+q^{15}\right)\left(\frac{1}{1-q^{3}}+\frac{q^{2}}{1-q^{2}}\right) \\
= & \frac{1+q^{15}}{1-q^{3}}+\frac{-q+q^{4}}{1-q^{3}}+\frac{q^{2}\left(1+q^{4}\right)}{1-q^{2}}+\frac{q^{2}\left(-q+q^{15}\right)}{1-q^{2}} \\
= & \frac{1+q^{15}}{1-q^{3}}+\frac{q^{2}+q^{6}}{1-q^{2}}-q-\left(q^{3}+q^{5}+\cdots+q^{15}\right),
\end{aligned}
$$

so we find that $s(n) \geq 0$ for $n \geq 16$. Moreover,

$$
\begin{aligned}
\sum_{\substack{n \geq 17 \\
n \text { odd }}} q^{n}\left(\frac{1}{1-q^{3}}+\frac{q^{2}}{1-q^{2}}\right) & =\sum_{n=8}^{\infty} q^{2 n+1}\left(1+\frac{q^{3}}{1-q^{3}}+\frac{q^{2}}{1-q^{2}}\right) \\
& =\frac{1}{1-q^{2}} \sum_{n=8}^{\infty} q^{2 n+1}+\frac{q^{3}}{1-q^{3}} \sum_{n=8}^{\infty} q^{2 n+1}
\end{aligned}
$$




$$
\begin{aligned}
& =\frac{q^{17}}{\left(1-q^{2}\right)^{2}}+\frac{q^{3}}{1-q^{3}} \sum_{n=8}^{\infty} q^{2 n+1} \\
& =\sum_{\substack{n \geq 17 \\
n \text { odd }}} \frac{n-15}{2} q^{n}+\frac{q^{3}}{1-q^{3}} \sum_{n=8}^{\infty} q^{2 n+1}
\end{aligned}
$$

and by (4.5), we deduce that $s(n) \geq(n-15) / 2$ when $n$ is odd and $n \geq 17$. Hence, by (4.4), we find that $f_{3}(n) \geq 0$ for $n \geq 16$ and $f_{3}(n) \geq(n-15) / 2$ when $n$ is odd and $n \geq 17$. Moreover, it can be checked that $f_{3}(n) \geq 0$ for $2 \leq n \leq 15$ and $n \neq 7$. Furthermore, $f_{3}(3)=f_{3}(5)=0, f_{3}(7)=-1$. Thus we complete the proof of Theorem 4.1 when $r=3$.

(4) When $r \geq 4$. Note that

$$
\begin{aligned}
\sum_{n=0}^{\infty} f_{r}(n) q^{n} & =\frac{1-q}{\left(q^{2} ; q\right)_{r-1}\left(q^{2} ; q\right)_{r}} \\
& =\frac{1}{\left(q^{2} ; q\right)_{r-1}} \sum_{n=0}^{\infty} d_{r+1}(n) q^{n}
\end{aligned}
$$

Since $d_{r+1}(0)=1$ and $d_{r+1}(1)=-1$, we have

$$
\begin{aligned}
\sum_{n=0}^{\infty} f_{r}(n) q^{n} & =\frac{1-q}{\left(q^{2} ; q\right)_{r-1}}+\frac{1}{\left(q^{2} ; q\right)_{r-1}} \sum_{n=2}^{\infty} d_{r+1}(n) q^{n} \\
& =\sum_{n=0}^{\infty} d_{r}(n) q^{n}+\frac{1}{\left(q^{2} ; q\right)_{r-1}} \sum_{n=2}^{\infty} d_{r+1}(n) q^{n}
\end{aligned}
$$

By Theorem 3.1 (7), we see that when $r \geq 7, d_{r}(n) \geq 0$ for $n \geq 2$ and $d_{r}(2 r+7) \geq 1$. Hence, by (4.7), we deduce that when $r \geq 7, f_{r}(n) \geq 0$ for $n \geq 2$ and $f_{r}(2 r+7) \geq 1$. Applying Theorem 3.1 (6) and (7), we see that $d_{6}(n) \geq 0$ for $n \geq 14$ and $d_{7}(n) \geq 0$ for $n \geq 2$, so we derive that $f_{6}(n) \geq 0$ for $n \geq 14$. It is easy to check that $f_{6}(n) \geq 0$ for $2 \leq n \leq 13$ and $f_{6}(19) \geq 1$. Thus we complete the proof of Theorem 4.1 when $r \geq 6$.

It remains to show that Theorem 4.1 holds when $r=4$ or $r=5$. Setting $r=4$ in (4.7), we see that

$$
\sum_{n=0}^{\infty} f_{4}(n) q^{n}=\sum_{n=0}^{\infty} d_{4}(n) q^{n}+\frac{1}{\left(q^{2} ; q\right)_{3}} \sum_{n=2}^{\infty} d_{5}(n) q^{n}
$$

From Theorem 3.1 (5), we see that $d_{5}(n) \geq 0$ for $n \geq 2$. Moreover, it is easy to see that $d_{5}(2)=1$. Hence

$$
\sum_{n=0}^{\infty} f_{4}(n) q^{n}=\sum_{n=0}^{\infty} d_{4}(n) q^{n}+\frac{q^{2}}{\left(q^{2} ; q\right)_{3}}+\frac{1}{\left(q^{2} ; q\right)_{3}} \sum_{n=3}^{\infty} d_{5}(n) q^{n}
$$




$$
=\sum_{n=0}^{\infty} d_{4}(n) q^{n}+\sum_{n=2}^{\infty} p_{4}(n-2) q^{n}+\frac{1}{\left(q^{2} ; q\right)_{3}} \sum_{n=3}^{\infty} d_{5}(n) q^{n} .
$$

By Theorem 3.1 (4), we have

$$
d_{4}(n) \geq-\left\lfloor\frac{n+11}{12}\right\rfloor
$$

and by Corollary 3.3 , we see that for $n \geq 14$,

$$
p_{4}(n-2) \geq\left\lfloor\frac{n-2}{6}\right\rfloor \text {. }
$$

Hence for $n \geq 14$,

$$
d_{4}(n)+p_{4}(n-2) \geq\left\lfloor\frac{n-2}{6}\right\rfloor-\left\lfloor\frac{n+11}{12}\right\rfloor \geq 0 .
$$

Furthermore, it is routine to check that $d_{4}(n)+p_{4}(n-2) \geq 0$ for $2 \leq n \leq 13$. Note that $d_{5}(n) \geq 0$ for $n \geq 2$, so by (4.8), we conclude that $f_{4}(n) \geq 0$ for $n \geq 2$. It is easy to check that $f_{4}(15) \geq 1$. Hence Theorem 4.1 is proved when $r=4$.

When $r=5$, by (4.1), we see that

$$
\begin{aligned}
\sum_{n=0}^{\infty} f_{5}(n) q^{n} & =\frac{1}{\left(q^{2} ; q\right)_{5}} \cdot \frac{1-q}{\left(q^{2} ; q\right)_{4}} \\
& =\frac{1}{\left(q^{2} ; q\right)_{5}} \sum_{n=0}^{\infty} d_{5}(n) q^{n} .
\end{aligned}
$$

Note that $d_{5}(0)=1$ and $d_{5}(1)=-1$, so we have

$$
\begin{aligned}
\sum_{n=0}^{\infty} f_{5}(n) q^{n} & =\frac{1-q}{\left(q^{2} ; q\right)_{5}}+\frac{1}{\left(q^{2} ; q\right)_{5}} \sum_{n=2}^{\infty} d_{5}(n) q^{n} \\
& =\sum_{n=0}^{\infty} d_{6}(n) q^{n}+\frac{1}{\left(q^{2} ; q\right)_{5}} \sum_{n=2}^{\infty} d_{5}(n) q^{n}
\end{aligned}
$$

Since $d_{6}(n) \geq 0$ for $n \geq 14$ and $d_{5}(n) \geq 0$ for $n \geq 2$, and by (4.9), we derive that $f_{5}(n) \geq 0$ for $n \geq 14$. It is trivial to check that $f_{5}(n) \geq 0$ for $2 \leq n \leq 13$ and $f_{5}(17) \geq 1$. Hence we arrive at $f_{5}(n) \geq 0$ for $n \geq 2$ and $f_{5}(17) \geq 1$. Thus, we complete the proof of Theorem 4.1 . 


\section{$5 \quad$ On $M(m, n) \geq M(m, n-1)$}

In this section, we will give a proof of Theorem 1.6 by means of Theorem 3.1.

Proof of Theorem 1.6. When $m=0$, by (2.2), we see that

$$
\sum_{n=0}^{\infty}(M(0, n)-M(0, n-1)) q^{n}=1-2 q+q^{2}+q^{3}+\sum_{k=2}^{\infty} \frac{q^{k^{2}+2 k}}{\left(q^{2} ; q\right)_{k-1}\left(q^{2} ; q\right)_{k-1}} .
$$

It yields that $M(0, n)-M(0, n-1) \geq 0$ for $n \geq 2$.

When $m=1,(2.2)$ becomes

$$
\sum_{n=0}^{\infty}(M(1, n)-M(1, n-1)) q^{n}=q-q^{2}+\frac{q^{5}}{1-q^{2}}+\sum_{k=2}^{\infty} \frac{q^{k^{2}+3 k+1}}{\left(q^{2} ; q\right)_{k-1}\left(q^{2} ; q\right)_{k}},
$$

which immediately implies that $M(1, n)-M(1, n-1) \geq 0$ for $n \geq 3$.

When $m \geq 2$, by (2.2), we have

$$
M(m, n)-M(m, n-1) \geq d_{m}(n-m)+p_{m+1}(n-2 m-3) .
$$

By Theorem $3.1(5)-(7)$, we see that $d_{m}(n-m) \geq 0$ for $m \geq 5$ and $n \geq m+2$ except for $(m, n)=(6,13)$ or $(m, n)=(6,19)$. By the definition of $p_{r}(n)$, we see that $p_{m+1}(n-2 m-3) \geq 0$ for $m \geq 1$. It follows from (5.1) that $M(m, n) \geq M(m, n-1)$ for $m \geq 5$ and $n \geq m+2$ except for $(m, n)=(6,13)$ or $(m, n)=(6,19)$. It is routine to check that $M(6,19)-M(6,18) \geq 0$ and $M(6,13)-M(6,12)=-1$. Since we only prove (1.8) holds when $n \geq 14$, we can omit the case $(m, n)=(6,13)$. Thus Theorem 1.6 is verified when $m \geq 5$.

From Theorem 3.1 (2)-(4), we see that when $2 \leq m \leq 4$ and $n \geq 4$,

$$
d_{m}(n-m) \geq-\left\lfloor\frac{n+8}{12}\right\rfloor \text {. }
$$

By Corollary 3.3 , we derive that when $2 \leq m \leq 4$ and $n \geq 2 m+15$,

$$
p_{m+1}(n-2 m-3) \geq\left\lfloor\frac{n-2 m-3}{6}\right\rfloor \text {. }
$$

It is easy to check that when $2 \leq m \leq 4$ and $n \geq 29$,

$$
\left\lfloor\frac{n-2 m-3}{6}\right\rfloor \geq\left\lfloor\frac{n+8}{12}\right\rfloor \text {. }
$$

So we derive that when $2 \leq m \leq 4$ and $n \geq 29$,

$$
M(m, n)-M(m, n-1) \geq d_{m}(n-m)+p_{m+1}(n-2 m-3) \geq 0 .
$$

Moreover, it can be checked that $M(m, n) \geq M(m, n-1)$ when $2 \leq m \leq 4$ and $14 \leq$ $n \leq 28$. So Theorem 1.6 is verified when $2 \leq m \leq 4$. Thus, we complete the proof of Theorem [1.6. 


\section{The generating function of $M(m-1, n)-M(m, n)$}

In this section, we will establish three expressions for the generating function of $M(m-$ $1, n)-M(m, n)$, which play a crucial role in the proof of Theorem 1.7. To this end, we first split the first summation in (2.4) into five summations as follows.

Lemma 6.1. For $m \geq 1$,

$$
\begin{aligned}
\sum_{k=1}^{\infty} \frac{q^{k(k+m-1)+2 k+m-1}}{(q ; q)_{k}\left(q^{2} ; q\right)_{k+m-2}}= & \frac{q^{2 m+2}}{\left(q^{2} ; q\right)_{m-1}}+\frac{q^{3 m+7}}{\left(1-q^{2}\right)\left(q^{2} ; q\right)_{m-1}} \\
& +\sum_{k=1}^{\infty} \frac{q^{k(k+m)+k+m-1}}{(q ; q)_{k-1}\left(q^{2} ; q\right)_{k+m-2}}+\sum_{k=3}^{\infty} \frac{q^{k(k+m)+2 k+m-1}}{\left(q^{2} ; q\right)_{k-1}\left(q^{2} ; q\right)_{k+m-4}} \\
& +\sum_{k=1}^{\infty} \frac{q^{k(k+m)+2 k+m}}{(q ; q)_{k}\left(q^{2} ; q\right)_{k+m-2}}+\sum_{k=3}^{\infty} \frac{q^{k(k+m)+3 k+2 m-3}}{\left(q^{2} ; q\right)_{k-1}\left(q^{2} ; q\right)_{k+m-3}} \\
& +\sum_{k=2}^{\infty} \frac{q^{k(k+m)+3 k+2 m-2}}{\left(q^{2} ; q\right)_{k-1}\left(q^{2} ; q\right)_{k+m-2}}
\end{aligned}
$$

Proof. It is clear that when $m \geq 1$,

$$
\sum_{k=1}^{\infty} \frac{q^{k(k+m-1)+2 k+m-1}}{(q ; q)_{k}\left(q^{2} ; q\right)_{k+m-2}}=\sum_{k=1}^{\infty} \frac{q^{k(k+m)+k+m-1}}{(q ; q)_{k-1}\left(q^{2} ; q\right)_{k+m-2}} \cdot \frac{1}{1-q^{k}}
$$

Obviously, when $k \geq 1$,

$$
\frac{1}{1-q^{k}}=1+\frac{q^{k}(1-q)}{1-q^{k}}+\frac{q^{k+1}}{1-q^{k}} .
$$

Substituting (6.3) into (6.2), we deduce that

$$
\begin{aligned}
\sum_{k=1}^{\infty} \frac{q^{k(k+m-1)+2 k+m-1}}{(q ; q)_{k}\left(q^{2} ; q\right)_{k+m-2}}= & \sum_{k=1}^{\infty} \frac{q^{k(k+m)+k+m-1}}{(q ; q)_{k-1}\left(q^{2} ; q\right)_{k+m-2}}+\sum_{k=1}^{\infty} \frac{q^{k(k+m)+2 k+m-1}}{\left(q^{2} ; q\right)_{k-1}\left(q^{2} ; q\right)_{k+m-2}} \\
& +\sum_{k=1}^{\infty} \frac{q^{k(k+m)+2 k+m}}{(q ; q)_{k}\left(q^{2} ; q\right)_{k+m-2}}
\end{aligned}
$$

Notice that

$$
\begin{aligned}
& \sum_{k=1}^{\infty} \frac{q^{k(k+m)+2 k+m-1}}{\left(q^{2} ; q\right)_{k-1}\left(q^{2} ; q\right)_{k+m-2}} \\
= & \frac{q^{2 m+2}}{\left(q^{2} ; q\right)_{m-1}}+\sum_{k=2}^{\infty} \frac{q^{k(k+m)+2 k+m-1}}{\left(q^{2} ; q\right)_{k-1}\left(q^{2} ; q\right)_{k+m-2}}
\end{aligned}
$$




$$
\begin{aligned}
= & \frac{q^{2 m+2}}{\left(q^{2} ; q\right)_{m-1}}+\sum_{k=2}^{\infty} \frac{q^{k(k+m)+2 k+m-1}}{\left(q^{2} ; q\right)_{k-1}\left(q^{2} ; q\right)_{k+m-3}}\left(1+\frac{q^{k+m-1}}{1-q^{k+m-1}}\right) \\
= & \frac{q^{2 m+2}}{\left(q^{2} ; q\right)_{m-1}}+\sum_{k=2}^{\infty} \frac{q^{k(k+m)+3 k+2 m-2}}{\left(q^{2} ; q\right)_{k-1}\left(q^{2} ; q\right)_{k+m-2}}+\sum_{k=2}^{\infty} \frac{q^{k(k+m)+2 k+m-1}}{\left(q^{2} ; q\right)_{k-1}\left(q^{2} ; q\right)_{k+m-3}} \\
= & \frac{q^{2 m+2}}{\left(q^{2} ; q\right)_{m-1}}+\sum_{k=2}^{\infty} \frac{q^{k(k+m)+3 k+2 m-2}}{\left(q^{2} ; q\right)_{k-1}\left(q^{2} ; q\right)_{k+m-2}}+\frac{q^{3 m+7}}{\left(1-q^{2}\right)\left(q^{2} ; q\right)_{m-1}} \\
& +\sum_{k=3}^{\infty} \frac{q^{k(k+m)+2 k+m-1}}{\left(q^{2} ; q\right)_{k-1}\left(q^{2} ; q\right)_{k+m-4}}\left(1+\frac{q^{k+m-2}}{1-q^{k+m-2}}\right) \\
= & \frac{q^{2 m+2}}{\left(q^{2} ; q\right)_{m-1}}+\frac{q^{3 m+7}}{\left(1-q^{2}\right)\left(q^{2} ; q\right)_{m-1}}+\sum_{k=2}^{\infty} \frac{q^{k(k+m)+3 k+2 m-2}}{\left(q^{2} ; q\right)_{k-1}\left(q^{2} ; q\right)_{k+m-2}} \\
& +\sum_{k=3}^{\infty} \frac{q^{k(k+m)+3 k+2 m-3}}{\left(q^{2} ; q\right)_{k-1}\left(q^{2} ; q\right)_{k+m-3}} \\
& +\sum_{k=3}^{\infty} \frac{q^{k(k+m)+2 k+m-1}}{\left(q^{2} ; q\right)_{k-1}\left(q^{2} ; q\right)_{k+m-4}} .
\end{aligned}
$$

Substituting (6.5) into (6.4), we are led to (6.1), and hence Lemma 6.1 follows.

The second summation in (2.4) can be split into the following five summations.

Lemma 6.2. For $m \geq 1$,

$$
\begin{aligned}
\sum_{k=1}^{\infty} \frac{q^{k(k+m)+2 k+m}}{(q ; q)_{k}\left(q^{2} ; q\right)_{k+m-1}}= & \sum_{k=1}^{\infty} \frac{q^{k(k+m)+2 k+m}}{(q ; q)_{k}\left(q^{2} ; q\right)_{k+m-2}}+\sum_{k=1}^{\infty} \frac{q^{k(k+m)+3 k+2 m}}{\left(q^{2} ; q\right)_{k-1}\left(q^{2} ; q\right)_{k+m-2}} \\
& +\sum_{k=1}^{\infty} \frac{q^{k(k+m)+3 k+2 m+1}}{(q ; q)_{k}\left(q^{2} ; q\right)_{k+m-1}}+\sum_{k=1}^{\infty} \frac{q^{k(k+m)+4 k+3 m}}{\left(q^{2} ; q\right)_{k-1}\left(q^{2} ; q\right)_{k+m-2}} \\
& +\sum_{k=1}^{\infty} \frac{q^{k(k+m)+5 k+4 m}}{\left(q^{2} ; q\right)_{k-1}\left(q^{2} ; q\right)_{k+m-1}}
\end{aligned}
$$

Proof. Clearly,

$$
\sum_{k=1}^{\infty} \frac{q^{k(k+m)+2 k+m}}{(q ; q)_{k}\left(q^{2} ; q\right)_{k+m-1}}=\sum_{k=1}^{\infty} \frac{q^{k(k+m)+2 k+m}}{(q ; q)_{k}\left(q^{2} ; q\right)_{k+m-2}} \cdot \frac{1}{1-q^{k+m}} .
$$

Moreover, one can easily check that the following identity holds:

$$
\frac{1}{1-q^{k+m}}=1+q^{k+m}(1-q)+\frac{q^{k+m+1}}{1-q^{k+m}}+q^{2 k+2 m}(1-q)+\frac{q^{3 k+3 m}(1-q)}{1-q^{k+m}} .
$$

Substituting (6.8) into (6.7), we obtain (6.6). This completes the proof. 
By Lemma 6.1 and Lemma 6.2, we obtain the first expression of the generating function of $M(m-1, n)-M(m, n)$ when $m \geq 1$.

Theorem 6.3. For $m \geq 1$,

$$
\begin{aligned}
& \sum_{n=0}^{\infty}(M(m-1, n)-M(m, n)) q^{n} \\
= & \frac{q^{m-1}(1-q)}{(q ; q)_{m-1}}-\frac{q^{m}}{\left(q^{2} ; q\right)_{m-1}}+\frac{q^{2 m+1}}{\left(q^{2} ; q\right)_{m-1}}+\frac{q^{2 m+2}}{\left(q^{2} ; q\right)_{m-1}} \\
& -\frac{q^{3 m+4}}{\left(q^{2} ; q\right)_{m-1}}+\frac{q^{3 m+7}}{\left(1-q^{2}\right)\left(q^{2} ; q\right)_{m-1}}-\frac{q^{5 m+6}}{\left(q^{2} ; q\right)_{m}} \\
& +\sum_{k=3}^{\infty} \frac{q^{k(k+m)+2 k+m-1}}{\left(q^{2} ; q\right)_{k-1}\left(q^{2} ; q\right)_{k+m-4}}+\sum_{k=2}^{\infty} \frac{q^{k(k+m)+3 k+2 m-2}}{\left(q^{3} ; q\right)_{k-2}\left(q^{2} ; q\right)_{k+m-2}} \\
& -\sum_{k=1}^{\infty} \frac{q^{k(k+m)+4 k+3 m}}{\left(q^{2} ; q\right)_{k-1}\left(q^{2} ; q\right)_{k+m-2}}+\sum_{k=2}^{\infty} \frac{q^{k(k+m)+5 k+3 m+1}\left(1-q^{m-1}\right)}{\left(q^{2} ; q\right)_{k}\left(q^{2} ; q\right)_{k+m-1}}
\end{aligned}
$$

Proof. Substituting (6.1) and (6.6) into (2.4), and by simplification, we get

$$
\begin{aligned}
& \sum_{n=0}^{\infty}(M(m-1, n)-M(m, n)) q^{n} \\
= & \left(\sum_{k=2}^{\infty} \frac{q^{k(k+m)+3 k+2 m-2}}{\left(q^{2} ; q\right)_{k-1}\left(q^{2} ; q\right)_{k+m-2}}-\sum_{k=1}^{\infty} \frac{q^{k(k+m)+3 k+2 m}}{\left(q^{2} ; q\right)_{k-1}\left(q^{2} ; q\right)_{k+m-2}}\right) \\
& +\left(\sum_{k=3}^{\infty} \frac{q^{k(k+m)+3 k+2 m-3}}{\left(q^{2} ; q\right)_{k-1}\left(q^{2} ; q\right)_{k+m-3}}-\sum_{k=1}^{\infty} \frac{q^{k(k+m)+5 k+4 m}}{\left(q^{2} ; q\right)_{k-1}\left(q^{2} ; q\right)_{k+m-1}}\right) \\
& +\left(\sum_{k=1}^{\infty} \frac{q^{k(k+m)+k+m-1}}{(q ; q)_{k-1}\left(q^{2} ; q\right)_{k+m-2}}-\sum_{k=1}^{\infty} \frac{q^{k(k+m)+3 k+2 m+1}}{(q ; q)_{k}\left(q^{2} ; q\right)_{k+m-1}}\right) \\
& +\sum_{k=3}^{\infty} \frac{q^{k(k+m)+2 k+m-1}}{\left(q^{2} ; q\right)_{k-1}\left(q^{2} ; q\right)_{k+m-4}}-\sum_{k=1}^{\infty} \frac{q^{k(k+m)+4 k+3 m}}{\left(q^{2} ; q\right)_{k-1}\left(q^{2} ; q\right)_{k+m-2}} \\
& +\frac{q^{m-1}(1-q)}{(q ; q)_{m-1}}-\frac{q^{m}}{\left(q^{2} ; q\right)_{m-1}}+\frac{q^{2 m+2}}{\left(q^{2} ; q\right)_{m-1}}+\frac{q^{3 m+7}}{\left(1-q^{2}\right)\left(q^{2} ; q\right)_{m-1}}
\end{aligned}
$$

Observe that

$$
\begin{aligned}
& \sum_{k=2}^{\infty} \frac{q^{k(k+m)+3 k+2 m-2}}{\left(q^{2} ; q\right)_{k-1}\left(q^{2} ; q\right)_{k+m-2}}-\sum_{k=1}^{\infty} \frac{q^{k(k+m)+3 k+2 m}}{\left(q^{2} ; q\right)_{k-1}\left(q^{2} ; q\right)_{k+m-2}} \\
= & -\frac{q^{3 m+4}}{\left(q^{2} ; q\right)_{m-1}}+\sum_{k=2}^{\infty} \frac{q^{k(k+m)+3 k+2 m-2}\left(1-q^{2}\right)}{\left(q^{2} ; q\right)_{k-1}\left(q^{2} ; q\right)_{k+m-2}}
\end{aligned}
$$




$$
=-\frac{q^{3 m+4}}{\left(q^{2} ; q\right)_{m-1}}+\sum_{k=2}^{\infty} \frac{q^{k(k+m)+3 k+2 m-2}}{\left(q^{3} ; q\right)_{k-2}\left(q^{2} ; q\right)_{k+m-2}}
$$

and

$$
\begin{aligned}
& \sum_{k=3}^{\infty} \frac{q^{k(k+m)+3 k+2 m-3}}{\left(q^{2} ; q\right)_{k-1}\left(q^{2} ; q\right)_{k+m-3}}-\sum_{k=1}^{\infty} \frac{q^{k(k+m)+5 k+4 m}}{\left(q^{2} ; q\right)_{k-1}\left(q^{2} ; q\right)_{k+m-1}} \\
= & \sum_{k=2}^{\infty} \frac{q^{k(k+m)+5 k+3 m+1}}{\left(q^{2} ; q\right)_{k}\left(q^{2} ; q\right)_{k+m-2}}-\sum_{k=1}^{\infty} \frac{q^{k(k+m)+5 k+4 m}}{\left(q^{2} ; q\right)_{k-1}\left(q^{2} ; q\right)_{k+m-1}} \\
= & -\frac{q^{5 m+6}}{\left(q^{2} ; q\right)_{m}}+\sum_{k=2}^{\infty} \frac{q^{k(k+m)+5 k+3 m+1}}{\left(q^{2} ; q\right)_{k}\left(q^{2} ; q\right)_{k+m-1}}\left(\left(1-q^{k+m}\right)-q^{m-1}\left(1-q^{k+1}\right)\right) \\
= & -\frac{q^{5 m+6}}{\left(q^{2} ; q\right)_{m}}+\sum_{k=2}^{\infty} \frac{q^{k(k+m)+5 k+3 m+1}\left(1-q^{m-1}\right)}{\left(q^{2} ; q\right)_{k}\left(q^{2} ; q\right)_{k+m-1}} .
\end{aligned}
$$

Moreover, it is easy to see that

$$
\sum_{k=1}^{\infty} \frac{q^{k(k+m)+k+m-1}}{(q ; q)_{k-1}\left(q^{2} ; q\right)_{k+m-2}}-\sum_{k=1}^{\infty} \frac{q^{k(k+m)+3 k+2 m+1}}{(q ; q)_{k}\left(q^{2} ; q\right)_{k+m-1}}=\frac{q^{2 m+1}}{\left(q^{2} ; q\right)_{m-1}}
$$

We then obtain (6.9) upon substituting (6.11), (6.12) and (6.13) into (6.10). This completes the proof.

When $m \geq 2$, we find that the generating function of $M(m-1, n)-M(m, n)$ in Theorem 6.3 can be further simplified as stated below.

Theorem 6.4. For $m \geq 2$,

$$
\begin{aligned}
& \sum_{n=0}^{\infty}(M(m-1, n)-M(m, n)) q^{n} \\
= & \frac{q^{m-1}}{\left(q^{2} ; q\right)_{m-2}}-\frac{q^{m}}{\left(q^{2} ; q\right)_{m-2}}-\frac{q^{2 m}}{\left(q^{3} ; q\right)_{m-2}}+\frac{q^{2 m+1}}{\left(q^{2} ; q\right)_{m-1}}-\frac{q^{3 m+4}}{\left(q^{2} ; q\right)_{m-1}} \\
& +\sum_{k=2}^{\infty} \frac{q^{k(k+m)+3 k+2 m-2}}{\left(q^{3} ; q\right)_{k-2}\left(q^{2} ; q\right)_{k+m-2}}+\sum_{k=1}^{\infty} \frac{q^{k(k+m)+4 k+2 m+2}\left(1-q^{m-2}\right)}{\left(q^{2} ; q\right)_{k}\left(q^{2} ; q\right)_{k+m-2}} \\
& +\sum_{k=1}^{\infty} \frac{q^{k(k+m)+5 k+3 m+1}\left(1-q^{m-1}\right)}{\left(q^{2} ; q\right)_{k}\left(q^{2} ; q\right)_{k+m-1}} .
\end{aligned}
$$

Proof. It is trivial to verify that when $m \geq 2$,

$$
\frac{q^{m}}{\left(q^{2} ; q\right)_{m-1}}=\frac{q^{m}}{\left(q^{2} ; q\right)_{m-2}}+\frac{q^{2 m}}{\left(q^{3} ; q\right)_{m-2}}+\frac{q^{2 m+2}}{\left(q^{2} ; q\right)_{m-1}}
$$


Hence, by Theorem 6.3, it suffices to show that

$$
\begin{aligned}
& \frac{q^{3 m+7}}{\left(1-q^{2}\right)\left(q^{2} ; q\right)_{m-1}}-\frac{q^{5 m+6}}{\left(q^{2} ; q\right)_{m}}+\sum_{k=3}^{\infty} \frac{q^{k(k+m)+2 k+m-1}}{\left(q^{2} ; q\right)_{k-1}\left(q^{2} ; q\right)_{k+m-4}} \\
& -\sum_{k=1}^{\infty} \frac{q^{k(k+m)+4 k+3 m}}{\left(q^{2} ; q\right)_{k-1}\left(q^{2} ; q\right)_{k+m-2}}+\sum_{k=2}^{\infty} \frac{q^{k(k+m)+5 k+3 m+1}\left(1-q^{m-1}\right)}{\left(q^{2} ; q\right)_{k}\left(q^{2} ; q\right)_{k+m-1}} \\
= & \sum_{k=1}^{\infty} \frac{q^{k(k+m)+4 k+2 m+2}\left(1-q^{m-2}\right)}{\left(q^{2} ; q\right)_{k}\left(q^{2} ; q\right)_{k+m-2}}+\sum_{k=1}^{\infty} \frac{q^{k(k+m)+5 k+3 m+1}\left(1-q^{m-1}\right)}{\left(q^{2} ; q\right)_{k}\left(q^{2} ; q\right)_{k+m-1}} .
\end{aligned}
$$

First, observe that

$$
\begin{aligned}
& \sum_{k=3}^{\infty} \frac{q^{k(k+m)+2 k+m-1}}{\left(q^{2} ; q\right)_{k-1}\left(q^{2} ; q\right)_{k+m-4}}-\sum_{k=1}^{\infty} \frac{q^{k(k+m)+4 k+3 m}}{\left(q^{2} ; q\right)_{k-1}\left(q^{2} ; q\right)_{k+m-2}} \\
= & \sum_{k=2}^{\infty} \frac{q^{k(k+m)+4 k+2 m+2}}{\left(q^{2} ; q\right)_{k}\left(q^{2} ; q\right)_{k+m-3}}-\sum_{k=1}^{\infty} \frac{q^{k(k+m)+4 k+3 m}}{\left(q^{2} ; q\right)_{k-1}\left(q^{2} ; q\right)_{k+m-2}} \\
= & -\frac{q^{4 m+5}}{\left(q^{2} ; q\right)_{m-1}}+\sum_{k=2}^{\infty} \frac{q^{k(k+m)+4 k+2 m+2}\left(1-q^{m-2}\right)}{\left(q^{2} ; q\right)_{k}\left(q^{2} ; q\right)_{k+m-2}} .
\end{aligned}
$$

On the other hand, we find that when $m \geq 2$,

$$
\begin{aligned}
& \frac{q^{3 m+7}}{\left(1-q^{2}\right)\left(q^{2} ; q\right)_{m-1}}-\frac{q^{4 m+5}}{\left(q^{2} ; q\right)_{m-1}}-\frac{q^{5 m+6}}{\left(q^{2} ; q\right)_{m}} \\
= & \frac{q^{3 m+7}}{\left(1-q^{2}\right)\left(q^{2} ; q\right)_{m-2}}\left(1+\frac{q^{m}}{1-q^{m}}\right)-\frac{q^{4 m+5}}{\left(q^{2} ; q\right)_{m-1}}-\frac{q^{5 m+6}}{\left(q^{2} ; q\right)_{m}} \\
= & \frac{q^{3 m+7}}{\left(1-q^{2}\right)\left(q^{2} ; q\right)_{m-2}}-\frac{q^{4 m+5}}{\left(q^{2} ; q\right)_{m-1}}+\frac{q^{4 m+7}}{\left(1-q^{2}\right)\left(q^{2} ; q\right)_{m-1}}-\frac{q^{5 m+6}}{\left(q^{2} ; q\right)_{m}} \\
= & \frac{q^{3 m+7}\left(1-q^{m-2}\right)}{\left(1-q^{2}\right)\left(q^{2} ; q\right)_{m-1}}+\frac{q^{4 m+7}\left(1-q^{m-1}\right)}{\left(1-q^{2}\right)\left(q^{2} ; q\right)_{m}} .
\end{aligned}
$$

Substituting (6.17) and (6.18) into the left-hand side of (6.16), we obtain the right-hand side of (6.16). This completes the proof of Theorem 6.3.

When $m \geq 3$, we could further simplify the generating function of $M(m-1, n)-$ $M(m, n)$ in Theorem 6.4 to obtain the following expression.

Theorem 6.5. For $m \geq 3$,

$$
\sum_{n=0}^{\infty}(M(m-1, n)-M(m, n)) q^{n}
$$




$$
\begin{aligned}
= & -q^{2 m}+q^{2 m+1}+q^{3 m+1}+\frac{q^{m-1}}{\left(q^{2} ; q\right)_{m-2}}-\frac{q^{m}}{\left(q^{2} ; q\right)_{m-2}}+\frac{q^{2 m+5}}{\left(q^{2} ; q\right)_{m-3}\left(1-q^{m}\right)} \\
& +\sum_{k=3}^{m} \frac{q^{2 k+2 m+1}}{\left(q^{k} ; q\right)_{m-k+1}}+\sum_{k=2}^{\infty} \frac{q^{k(k+m)+3 k+2 m-2}}{\left(q^{3} ; q\right)_{k-2}\left(q^{2} ; q\right)_{k+m-2}}+\sum_{k=1}^{\infty} \frac{q^{k(k+m)+4 k+2 m+2}\left(1-q^{m-2}\right)}{\left(q^{2} ; q\right)_{k}\left(q^{2} ; q\right)_{k+m-2}} \\
& +\sum_{k=1}^{\infty} \frac{q^{k(k+m)+5 k+3 m+1}}{\left(q^{2} ; q\right)_{k}\left(q^{2} ; q\right)_{m-3}\left(q^{m} ; q\right)_{k+1}} .
\end{aligned}
$$

Proof. From Theorem 6.4, it suffices to show that when $m \geq 3$,

$$
\begin{aligned}
& \frac{q^{2 m+1}}{\left(q^{2} ; q\right)_{m-1}}-\frac{q^{2 m}}{\left(q^{3} ; q\right)_{m-2}}-\frac{q^{3 m+4}}{\left(q^{2} ; q\right)_{m-1}} \\
= & -q^{2 m}+q^{2 m+1}+q^{3 m+1}+\frac{q^{2 m+5}}{\left(q^{2} ; q\right)_{m-3}\left(1-q^{m}\right)}+\sum_{k=3}^{m} \frac{q^{2 k+2 m+1}}{\left(q^{k} ; q\right)_{m-k+1}} .
\end{aligned}
$$

In light of (3.12), we see that

$$
\begin{aligned}
\frac{q^{2 m+1}}{\left(q^{2} ; q\right)_{m-1}} & =q^{2 m+1}+\sum_{k=2}^{m} \frac{q^{2 m+1+k}}{\left(q^{k} ; q\right)_{m-k+1}} \\
& =q^{2 m+1}+\sum_{k=2}^{m} \frac{q^{2 m+1+k}\left(1-q^{k}+q^{k}\right)}{\left(q^{k} ; q\right)_{m-k+1}} \\
& =q^{2 m+1}+\sum_{k=2}^{m} \frac{q^{k+2 m+1}}{\left(q^{k+1} ; q\right)_{m-k}}+\sum_{k=2}^{m} \frac{q^{2 k+2 m+1}}{\left(q^{k} ; q\right)_{m-k+1}}
\end{aligned}
$$

Using the same argument as in the proof of (3.12), we deduce that for $m \geq 3$,

$$
\frac{q^{2 m}}{\left(q^{3} ; q\right)_{m-2}}=q^{2 m}+\sum_{k=3}^{m} \frac{q^{k+2 m}}{\left(q^{k} ; q\right)_{m-k+1}}=q^{2 m}+\sum_{k=2}^{m-1} \frac{q^{k+2 m+1}}{\left(q^{k+1} ; q\right)_{m-k}}
$$

Substituting (6.21) and (6.22) into the left-hand side of (6.20), we obtain

$$
\begin{aligned}
& \frac{q^{2 m+1}}{\left(q^{2} ; q\right)_{m-1}}-\frac{q^{2 m}}{\left(q^{3} ; q\right)_{m-2}}-\frac{q^{3 m+4}}{\left(q^{2} ; q\right)_{m-1}} \\
= & -q^{2 m}+q^{2 m+1}+q^{3 m+1}+\sum_{k=3}^{m} \frac{q^{2 k+2 m+1}}{\left(q^{k} ; q\right)_{m-k+1}}+\frac{q^{2 m+5}}{\left(q^{2} ; q\right)_{m-1}}-\frac{q^{3 m+4}}{\left(q^{2} ; q\right)_{m-1}} \\
= & -q^{2 m}+q^{2 m+1}+q^{3 m+1}+\sum_{k=3}^{m} \frac{q^{2 k+2 m+1}}{\left(q^{k} ; q\right)_{m-k+1}}+\frac{q^{2 m+5}}{\left(q^{2} ; q\right)_{m-3}\left(1-q^{m}\right)},
\end{aligned}
$$

which is equal to the right-hand side of (6.20). Thus, we complete the proof of Theorem 6.5 . 


\section{On $M(1, n) \geq M(2, n)$}

In the following three sections, we will give a proof of Theorem 1.7. In this section, we will show that Theorem 1.7 holds when $m=2$. In Section 8 , we will prove that Theorem 1.7 holds when $m \geq 3$. Section 9 is devoted to the proof of Theorem 1.7 when $m=1$. As stated in Section 2, the proof of Theorem 1.7 when $m=1$ is the most complicated, so we put the proof of the case $m=1$ at the end of the proof of the whole theorem.

Proof of Theorem 1.7 for $m=2$. Setting $m=2$ in Theorem 6.4, we have

$$
\begin{aligned}
& \sum_{n=0}^{\infty}(M(1, n)-M(2, n)) q^{n} \\
= & q-q^{2}-q^{4}+\frac{q^{5}}{1-q^{2}}-\frac{q^{10}}{1-q^{2}}+\sum_{k=2}^{\infty} \frac{q^{k^{2}+5 k+2}}{\left(q^{3} ; q\right)_{k-2}\left(q^{2} ; q\right)_{k}} \\
& +\sum_{k=1}^{\infty} \frac{q^{k^{2}+7 k+7}(1-q)}{\left(q^{2} ; q\right)_{k}\left(q^{2} ; q\right)_{k+1}} .
\end{aligned}
$$

Observe that

$$
\begin{aligned}
& \sum_{k=2}^{\infty} \frac{q^{k^{2}+5 k+2}}{\left(q^{3} ; q\right)_{k-2}\left(q^{2} ; q\right)_{k}}-\frac{q^{10}}{1-q^{2}} \\
= & \sum_{k=3}^{\infty} \frac{q^{k^{2}+5 k+2}}{\left(q^{3} ; q\right)_{k-2}\left(q^{2} ; q\right)_{k}}+\frac{q^{16}}{\left(1-q^{2}\right)\left(1-q^{3}\right)}-\frac{q^{10}}{1-q^{2}} \\
= & \sum_{k=3}^{\infty} \frac{q^{k^{2}+5 k+2}}{\left(q^{3} ; q\right)_{k-2}\left(q^{2} ; q\right)_{k}}+\frac{q^{19}}{\left(1-q^{2}\right)\left(1-q^{3}\right)}-q^{10}-q^{12}-q^{14} .
\end{aligned}
$$

Define

$$
\sum_{n=0}^{\infty} T_{2}(n) q^{n}:=\sum_{k=1}^{\infty} \frac{q^{k^{2}+7 k+7}(1-q)}{\left(q^{2} ; q\right)_{k}\left(q^{2} ; q\right)_{k+1}}
$$

and by (7.1) and (7.2), we find that for $n \geq 15$,

$$
M(1, n)-M(2, n) \geq T_{2}(n) .
$$

Hence it suffices to show that $T_{2}(n) \geq 0$ when $n \geq 44$.

By (4.1) and (7.3), we find that $T_{2}(n)$ can be expressed in terms of $f_{r}(n)$ as follows.

$$
\sum_{n=0}^{\infty} T_{2}(n) q^{n}=\sum_{k=1}^{\infty} \frac{q^{k^{2}+7 k+7}(1-q)}{\left(q^{2} ; q\right)_{k}\left(q^{2} ; q\right)_{k+1}}=\sum_{k=1}^{\infty} q^{k^{2}+7 k+7} \sum_{n=0}^{\infty} f_{k+1}(n) q^{n} .
$$


Define

$$
\begin{aligned}
\sum_{n=0}^{\infty} R(n) q^{n} & :=q^{15} \sum_{n=0}^{\infty} f_{2}(n) q^{n}+q^{25} \sum_{n=0}^{\infty} f_{3}(n) q^{n} \\
\sum_{n=0}^{\infty} S(n) q^{n} & :=\sum_{k=3}^{\infty} q^{k^{2}+7 k+7} \sum_{n=0}^{\infty} f_{k+1}(n) q^{n} .
\end{aligned}
$$

By (7.5), we find that for $n \geq 0$,

$$
T_{2}(n)=R(n)+S(n) .
$$

We will investigate the nonnegativity of $R(n)$ and $S(n)$ respectively.

By Theorem 4.1 (2), we see that

$$
\begin{aligned}
q^{15} \sum_{n=0}^{\infty} f_{2}(n) q^{n} & =q^{15}\left(\sum_{m=0}^{\infty} f_{2}(2 m) q^{2 m}-\sum_{m=0}^{\infty}\left\lceil\frac{2 m+1}{6}\right\rceil q^{2 m+1}\right) \\
& =\sum_{m=7}^{\infty} f_{2}(2 m-14) q^{2 m+1}-\sum_{m=8}^{\infty}\left\lceil\frac{2 m-15}{6}\right\rceil q^{2 m} .
\end{aligned}
$$

From Theorem 4.1 (3), we have

$$
\begin{aligned}
q^{25} \sum_{n=0}^{\infty} f_{3}(n) q^{n} & =q^{25}\left(1-q-q^{7}+\sum_{m=4}^{\infty} f_{3}(2 m+1) q^{2 m+1}+\sum_{m=1}^{\infty} f_{3}(2 m) q^{2 m}\right) \\
& =q^{25}-q^{26}-q^{32}+\sum_{m=17}^{\infty} f_{3}(2 m-25) q^{2 m}+\sum_{m=13}^{\infty} f_{3}(2 m-24) q^{2 m+1} .
\end{aligned}
$$

Combining (17.7) and (7.8), we find that

$$
\begin{aligned}
\sum_{n=15}^{\infty} R(n) q^{n}= & q^{25}-q^{26}-q^{32}-\sum_{m=8}^{20}\left\lceil\frac{2 m-15}{6}\right\rceil q^{2 m} \\
& +\sum_{m=7}^{\infty} f_{2}(2 m-14) q^{2 m+1}+\sum_{m=17}^{20} f_{3}(2 m-25) q^{2 m} \\
& +\sum_{m=13}^{\infty} f_{3}(2 m-24) q^{2 m+1} \\
& +\sum_{m=21}^{\infty}\left(f_{3}(2 m-25)-\left\lceil\frac{2 m-15}{6}\right\rceil\right) q^{2 m} .
\end{aligned}
$$

By Theorem 4.1 (2) and (3), we see that $f_{2}(2 m-14) \geq 0$ when $m \geq 7$ and $f_{3}(2 m-24) \geq 0$ when $m \geq 13$. It yields that when $m \geq 7$,

$$
R(2 m+1) \geq 0 \text {. }
$$


Using Theorem 4.1 (3), we see that $f_{3}(2 m-25) \geq m-20$ for $m \geq 21$. It follows that when $m \geq 27$,

$$
f_{3}(2 m-25)-\left\lceil\frac{2 m-15}{6}\right\rceil \geq m-20-\left\lceil\frac{2 m-15}{6}\right\rceil \geq 0 .
$$

Hence we derive that when $m \geq 27$,

$$
R(2 m) \geq 0
$$

Combining (7.9) and (7.10), we derive that when $n \geq 54$,

$$
R(n) \geq 0 \text {. }
$$

It can be checked that $R(n) \geq 0$ for $44 \leq n \leq 53$. Thus we show that $R(n) \geq 0$ for $n \geq 44$.

We proceed to investigate the nonnegativity of $S(n)$. Observe that

$$
\begin{aligned}
\sum_{n=0}^{\infty} S(n) q^{n}= & \sum_{k=3}^{\infty} q^{k^{2}+7 k+7} \sum_{n=0}^{\infty} f_{k+1}(n) q^{n} \\
= & \sum_{k=3}^{\infty} q^{k^{2}+7 k+7}\left(1-q+f_{k+1}(2 k+9) q^{2 k+9}+\sum_{\substack{n \geq 2 \\
n \neq 2 k+9}} f_{k+1}(n) q^{n}\right) \\
= & \sum_{k=3}^{\infty} q^{k^{2}+7 k+7}\left(1+\sum_{\substack{n \geq 2 \\
n \neq 2 k+9}} f_{k+1}(n) q^{n}\right) \\
& +\sum_{k=3}^{\infty} f_{k+1}(2 k+9) q^{k^{2}+9 k+16}-\sum_{k=3}^{\infty} q^{k^{2}+7 k+8}
\end{aligned}
$$

It is clear to see that

$$
\begin{aligned}
& \sum_{k=3}^{\infty} f_{k+1}(2 k+9) q^{k^{2}+9 k+16}-\sum_{k=3}^{\infty} q^{k^{2}+7 k+8} \\
= & \sum_{k=4}^{\infty} f_{k}(2 k+7) q^{k^{2}+7 k+8}-\sum_{k=3}^{\infty} q^{k^{2}+7 k+8} \\
= & -q^{38}+\sum_{k=4}^{\infty}\left(f_{k}(2 k+7)-1\right) q^{k^{2}+7 k+8} .
\end{aligned}
$$

Substituting (7.12) into (7.11), we obtain

$$
\sum_{n=0}^{\infty} S(n) q^{n}=-q^{38}+\sum_{k=3}^{\infty} q^{k^{2}+7 k+7}\left(1+\sum_{\substack{n \geq 2 \\ n \neq 2 k+9}} f_{k+1}(n) q^{n}\right)
$$




$$
+\sum_{k=4}^{\infty}\left(f_{k}(2 k+7)-1\right) q^{k^{2}+7 k+8}
$$

From Theorem 4.1 (4), we see that $f_{k}(n) \geq 0$ for $k \geq 4$ and $n \geq 2$ and $f_{k}(2 k+7) \geq 1$. It follows that $S(n) \geq 0$ when $n \geq 39$. Thus, by (17.6), we conclude that $T_{2}(n) \geq 0$ for $n \geq 44$, and so $M(1, n)-M(2, n) \geq 0$ for $n \geq 44$. Hence we complete the proof of Theorem 1.7 for $m=2$.

\section{On $M(m-1, n) \geq M(m, n)$ when $m \geq 3$}

In this section, we prove that Theorem 1.7 holds when $m \geq 3$ by means of Theorem 3.1 , Corollary 3.3 and Theorem 6.5.

Proof of Theorem 1.7 for $m \geq 3$. Define

$$
\begin{aligned}
\sum_{n=0}^{\infty} T_{m}(n) q^{n}:= & -q^{2 m}+q^{2 m+1}+q^{3 m+1}+q^{m-1} \frac{1-q}{\left(q^{2} ; q\right)_{m-2}} \\
& +\sum_{k=2}^{\infty} \frac{q^{k(k+m)+3 k+2 m-2}}{\left(q^{3} ; q\right)_{k-2}\left(q^{2} ; q\right)_{k+m-2}}+\sum_{k=1}^{\infty} \frac{q^{k(k+m)+4 k+2 m+2}\left(1-q^{m-2}\right)}{\left(q^{2} ; q\right)_{k}\left(q^{2} ; q\right)_{k+m-2}}
\end{aligned}
$$

From Theorem 6.5, it can be seen that for $m \geq 3$ and $n \geq 0$,

$$
M(m-1, n)-M(m, n) \geq T_{m}(n) .
$$

Define

$$
\sum_{n=0}^{\infty} U_{m}(n) q^{n}:=-q^{2 m}+q^{2 m+1}+q^{3 m+1}+q^{m-1} \frac{1-q}{\left(q^{2} ; q\right)_{m-2}}+\frac{q^{4 m+8}}{\left(q^{2} ; q\right)_{m}}
$$

SO

$$
\sum_{n=0}^{\infty}\left(T_{m}(n)-U_{m}(n)\right) q^{n}=\sum_{k=3}^{\infty} \frac{q^{k(k+m)+3 k+2 m-2}}{\left(q^{3} ; q\right)_{k-2}\left(q^{2} ; q\right)_{k+m-2}}+\sum_{k=1}^{\infty} \frac{q^{k(k+m)+4 k+2 m+2}\left(1-q^{m-2}\right)}{\left(q^{2} ; q\right)_{k}\left(q^{2} ; q\right)_{k+m-2}} .
$$

When $m=3$, observe that

$$
\sum_{k=1}^{\infty} \frac{q^{k(k+m)+4 k+2 m+2}\left(1-q^{m-2}\right)}{\left(q^{2} ; q\right)_{k}\left(q^{2} ; q\right)_{k+m-2}}=\sum_{k=1}^{\infty} \frac{q^{k^{2}+7 k+8}(1-q)}{\left(q^{2} ; q\right)_{k}\left(q^{2} ; q\right)_{k+1}}=\sum_{n=0}^{\infty} T_{2}(n) q^{n+1},
$$

where $T_{2}(n)$ is defined in (7.3). From the proof of Theorem 1.7 for $m=2$, we see that $T_{2}(n) \geq 0$ for $n \geq 44$. Moreover, it can be checked that $T_{2}(43) \geq 0$. 
When $m \geq 4$,

$$
\sum_{k=1}^{\infty} \frac{q^{k(k+m)+4 k+2 m+2}\left(1-q^{m-2}\right)}{\left(q^{2} ; q\right)_{k}\left(q^{2} ; q\right)_{k+m-2}}=\sum_{k=1}^{\infty} \frac{q^{k(k+m)+4 k+2 m+2}}{\left(q^{2} ; q\right)_{k}\left(q^{2} ; q\right)_{m-4}\left(q^{m-1} ; q\right)_{k+1}}
$$

which obviously has nonnegative power series coefficients. So in either case (8.4) implies that for $m \geq 3$ and $n \geq 44$,

$$
T_{m}(n) \geq U_{m}(n)
$$

We proceed to establish the nonnegativity of $U_{m}(n)$ when $m \geq 3$. Observe that

$$
\begin{aligned}
\sum_{n=0}^{\infty} U_{m}(n) q^{n}= & -q^{2 m}+q^{2 m+1}+q^{3 m+1}+q^{m-1}\left(1-q+\sum_{n=2}^{\infty} d_{m-1}(n) q^{n}\right) \\
& +\sum_{n=0}^{\infty} p_{m+1}(n) q^{n+4 m+8} \\
= & q^{m-1}-q^{m}+q^{2 m+1}+q^{3 m+1}+\sum_{\substack{n \geq 2 \\
n \neq m+1}} d_{m-1}(n) q^{n+m-1} \\
& +\left(d_{m-1}(m+1)-1\right) q^{2 m}+\sum_{n=0}^{\infty} p_{m+1}(n) q^{n+4 m+8} .
\end{aligned}
$$

There are two cases:

Case 1. When $3 \leq m \leq 7$, from (8.6), we see that for $n \geq 44$,

$$
U_{m}(n)=d_{m-1}(n-m+1)+p_{m+1}(n-4 m-8) .
$$

By Theorem 3.1 (2)-(6), we see that when $3 \leq m \leq 7$ and $n \geq m-1$,

$$
d_{m-1}(n-m+1) \geq-\left\lfloor\frac{n-m+12}{12}\right\rfloor .
$$

By Corollary 3.3 , we have for $n \geq 4 m+8$,

$$
p_{m+1}(n-4 m-8) \geq\left\lfloor\frac{n-4 m-8}{6}\right\rfloor .
$$

Thus by (8.7), we derive that when $3 \leq m \leq 7$ and $n \geq 72$,

$$
U_{m}(n) \geq\left\lfloor\frac{n-4 m-8}{6}\right\rfloor-\left\lfloor\frac{n-m+12}{12}\right\rfloor \geq 0 .
$$

It is trivial to check that $U_{m}(n) \geq 0$ for $44 \leq n \leq 71$. So we are led to $U_{m}(n) \geq 0$ for $n \geq 44$ and $3 \leq m \leq 7$. 
Case 2. When $m \geq 8$, from (8.6), we derive that when $n \geq m+1$ and $n \neq 2 m$,

$$
U_{m}(n) \geq d_{m-1}(n-m+1)+p_{m+1}(n-4 m-8) .
$$

When $n=2 m$,

$$
U_{m}(n)=d_{m-1}(m+1)-1 .
$$

By Theorem 3.1 (7), we find that $d_{m-1}(n-m+1)+p_{m+1}(n-4 m-8) \geq 0$ and $d_{m-1}(m+1) \geq$ 1 when $m \geq 8$ and $n \geq m+1$. Hence by (8.8) and (8.9), we derive that $U_{m}(n) \geq 0$ when $m \geq 8$ and $n \geq m+1$. So $U_{m}(n) \geq 0$ when $m \geq 3$ and $n \geq 44$. Hence it follows from (8.2) and (8.5) that $M(m-1, n)-M(m, n) \geq 0$ for $m \geq 3$ and $n \geq 44$. This completes the proof of Theorem 1.7 when $m \geq 3$.

\section{On $M(0, n) \geq M(1, n)$}

In the section, we finish the proof of Theorem 1.7 by showing that $M(0, n) \geq M(1, n)$ for $n \geq 44$. As has already been mentioned in Section 2, the proof of $M(0, n) \geq M(1, n)$ for $n \geq 44$ is the most complicated. Setting $m=1$ in Theorem 6.3, we find that

$$
\begin{aligned}
& \sum_{n=0}^{\infty}(M(0, n)-M(1, n)) q^{n} \\
= & 1-2 q+q^{3}+q^{4}-q^{7}+\frac{q^{10}}{1-q^{2}}-\frac{q^{11}}{1-q^{2}} \\
& +\sum_{k=3}^{\infty} \frac{q^{k^{2}+3 k}}{\left(q^{2} ; q\right)_{k-1}\left(q^{2} ; q\right)_{k-3}}+\sum_{k=2}^{\infty} \frac{q^{k^{2}+4 k}}{\left(q^{3} ; q\right)_{k-2}\left(q^{2} ; q\right)_{k-1}} \\
& -\sum_{k=1}^{\infty} \frac{q^{k^{2}+5 k+3}}{\left(q^{2} ; q\right)_{k-1}\left(q^{2} ; q\right)_{k-1}} .
\end{aligned}
$$

To show that Theorem 1.7 holds when $m=1$, we next aim to show that the following generating function of $M(0, n)-M(1, n)$ holds.

Theorem 9.1. We have

$$
\begin{aligned}
& \sum_{n=0}^{\infty}(M(0, n)-M(1, n)) q^{n} \\
= & 1-2 q+q^{3}+q^{4}-q^{7}-q^{9}+q^{18}+\frac{q^{10}}{1-q^{2}}-\frac{q^{11}}{1-q^{2}} \\
& +\frac{q^{12}}{1-q^{2}}-\frac{q^{17}}{1-q^{2}}-\frac{q^{19}}{1-q^{2}}+\frac{q^{20}}{1-q^{2}}+\frac{q^{21}}{1-q^{2}}-\frac{q^{23}}{\left(1-q^{2}\right)^{2}}
\end{aligned}
$$




$$
\begin{aligned}
& +\frac{q^{24}}{\left(q^{2} ; q\right)_{2}}+\frac{q^{28}}{\left(1-q^{2}\right)^{2}}-\frac{q^{38}}{\left(1-q^{3}\right)^{2}}+\sum_{k=3}^{\infty} \frac{q^{k^{2}+5 k}}{\left(q^{4} ; q\right)_{k-3}\left(q^{2} ; q\right)_{k-1}} \\
& +\sum_{k=3}^{\infty} \frac{q^{k^{2}+5 k}}{\left(q^{3} ; q\right)_{k-2}\left(q^{2} ; q\right)_{k-3}}+\sum_{k=3}^{\infty} \frac{q^{k^{2}+5 k+2}}{\left(1-q^{2}\right)\left(q^{4} ; q\right)_{k-3}\left(q^{2} ; q\right)_{k-3}} \\
& +\sum_{k=3}^{\infty} \sum_{i=0}^{\infty} \frac{q^{k^{2}+6 k+5+(k-1) i}\left(1-q^{i+2}\right)}{\left(q^{2} ; q\right)_{k-1}\left(q^{2} ; q\right)_{k-3}}+\sum_{k=3}^{\infty} \sum_{i=0}^{\infty} \frac{q^{k^{2}+9 k+8+i k}\left(1-q^{i+8}\right)}{\left(q^{2} ; q\right)_{k-2}\left(q^{3} ; q\right)_{k-2}\left(1-q^{k+1}\right)} .
\end{aligned}
$$

To obtain Theorem 9.1, we are required to further expand three summations in (9.1).

Lemma 9.2. We have

$$
\begin{aligned}
& \sum_{k=3}^{\infty} \frac{q^{k^{2}+3 k}}{\left(q^{2} ; q\right)_{k-1}\left(q^{2} ; q\right)_{k-3}} \\
= & \sum_{k=3}^{\infty} \frac{q^{k^{2}+3 k}}{\left(q^{2} ; q\right)_{k-3}\left(q^{2} ; q\right)_{k-3}}+\sum_{k=3}^{\infty} \frac{q^{k^{2}+4 k-1}}{\left(q^{2} ; q\right)_{k-2}\left(q^{2} ; q\right)_{k-3}}+\sum_{k=3}^{\infty} \frac{q^{k^{2}+4 k}}{\left(q^{2} ; q\right)_{k-2}\left(q^{2} ; q\right)_{k-3}} \\
& +\sum_{k=3}^{\infty} \frac{q^{k^{2}+5 k}}{\left(q^{2} ; q\right)_{k-1}\left(q^{2} ; q\right)_{k-3}} .
\end{aligned}
$$

Proof. It is clear that

$$
\sum_{k=3}^{\infty} \frac{q^{k^{2}+3 k}}{\left(q^{2} ; q\right)_{k-1}\left(q^{2} ; q\right)_{k-3}}=\sum_{k=3}^{\infty} \frac{q^{k^{2}+3 k}}{\left(q^{2} ; q\right)_{k-2}\left(q^{2} ; q\right)_{k-3}} \cdot \frac{1}{1-q^{k}} .
$$

Obviously, when $k \geq 1$,

$$
\frac{1}{1-q^{k}}=\left(1-q^{k-1}\right)+q^{k-1}+q^{k}+\frac{q^{2 k}}{1-q^{k}},
$$

so we can obtain (9.3) by substituting (9.5) into (9.4).

Lemma 9.3. We have

$$
\begin{aligned}
& \sum_{k=2}^{\infty} \frac{q^{k^{2}+4 k}}{\left(q^{3} ; q\right)_{k-2}\left(q^{2} ; q\right)_{k-1}} \\
= & \frac{q^{12}}{1-q^{2}}+\sum_{k=3}^{\infty} \frac{q^{k^{2}+4 k}}{\left(q^{3} ; q\right)_{k-3}\left(q^{2} ; q\right)_{k-2}}+\sum_{k=3}^{\infty} \frac{q^{k^{2}+5 k}}{\left(q^{3} ; q\right)_{k-2}\left(q^{2} ; q\right)_{k-1}} \\
& +\sum_{k=3}^{\infty} \frac{q^{k^{2}+5 k}}{\left(q^{3} ; q\right)_{k-3}\left(q^{2} ; q\right)_{k-1}} .
\end{aligned}
$$


Proof. Observe that

$$
\sum_{k=2}^{\infty} \frac{q^{k^{2}+4 k}}{\left(q^{3} ; q\right)_{k-2}\left(q^{2} ; q\right)_{k-1}}=\frac{q^{12}}{1-q^{2}}+\sum_{k=3}^{\infty} \frac{q^{k^{2}+4 k}}{\left(q^{3} ; q\right)_{k-3}\left(q^{2} ; q\right)_{k-1}} \cdot \frac{1}{1-q^{k}}
$$

Clearly, when $k \geq 1$,

$$
\frac{1}{1-q^{k}}=\left(1-q^{k}\right)+\frac{q^{k}}{1-q^{k}}+q^{k}
$$

Substituting (9.8) into (9.7), we obtain (9.6).

Lemma 9.4. We have

$$
\begin{aligned}
& \sum_{k=1}^{\infty} \frac{q^{k^{2}+5 k+3}}{\left(q^{2} ; q\right)_{k-1}\left(q^{2} ; q\right)_{k-1}} \\
= & q^{9}+\frac{q^{19}}{1-q^{2}}+\frac{q^{23}}{\left(1-q^{2}\right)^{2}}+\sum_{k=2}^{\infty} \frac{q^{k^{2}+5 k+3}}{\left(q^{2} ; q\right)_{k-1}\left(q^{3} ; q\right)_{k-2}}+\sum_{k=3}^{\infty} \frac{q^{k^{2}+5 k+5}}{\left(q^{2} ; q\right)_{k-1}\left(q^{2} ; q\right)_{k-3}} \\
& +\sum_{k=3}^{\infty} \frac{q^{k^{2}+6 k+4}}{\left(q^{2} ; q\right)_{k-1}\left(q^{2} ; q\right)_{k-2}}+\sum_{k=2}^{\infty} \frac{q^{k^{2}+6 k+5}}{\left(q^{2} ; q\right)_{k-1}\left(q^{3} ; q\right)_{k-2}}+\sum_{k=3}^{\infty} \frac{q^{k^{2}+6 k+7}}{\left(q^{2} ; q\right)_{k-1}\left(q^{2} ; q\right)_{k-3}\left(1-q^{k}\right)} \\
& +\sum_{k=3}^{\infty} \frac{q^{k^{2}+7 k+6}\left(1+q^{2}\right)}{\left(q^{2} ; q\right)_{k-1}\left(q^{3} ; q\right)_{k-2}}+\sum_{k=3}^{\infty} \frac{q^{k^{2}+7 k+10}}{\left(q^{2} ; q\right)_{k-1}\left(q^{2} ; q\right)_{k-1}} .
\end{aligned}
$$

Proof. Clearly,

$$
\sum_{k=1}^{\infty} \frac{q^{k^{2}+5 k+3}}{\left(q^{2} ; q\right)_{k-1}\left(q^{2} ; q\right)_{k-1}}=q^{9}+\sum_{k=2}^{\infty} \frac{q^{k^{2}+5 k+3}}{\left(q^{2} ; q\right)_{k-1}\left(q^{3} ; q\right)_{k-2}} \cdot \frac{1}{1-q^{2}} .
$$

It is trivial to check that

$$
\frac{1}{1-q^{2}}=1+\frac{q^{2}\left(1-q^{k}\right)}{1-q^{2}}+\frac{q^{k+2}}{1-q^{2}} .
$$

Substituting (9.11) into (9.10), we have

$$
\begin{aligned}
\sum_{k=1}^{\infty} \frac{q^{k^{2}+5 k+3}}{\left(q^{2} ; q\right)_{k-1}\left(q^{2} ; q\right)_{k-1}}= & q^{9}+\sum_{k=2}^{\infty} \frac{q^{k^{2}+5 k+3}}{\left(q^{2} ; q\right)_{k-1}\left(q^{3} ; q\right)_{k-2}}+\sum_{k=2}^{\infty} \frac{q^{k^{2}+5 k+5}}{\left(q^{2} ; q\right)_{k-1}\left(q^{2} ; q\right)_{k-2}} \\
& +\sum_{k=2}^{\infty} \frac{q^{k^{2}+6 k+5}}{\left(q^{2} ; q\right)_{k-1}\left(q^{2} ; q\right)_{k-1}} .
\end{aligned}
$$

We next transform the third and the fourth terms in (9.12) respectively. First, we rewrite the third term as follows.

$$
\sum_{k=2}^{\infty} \frac{q^{k^{2}+5 k+5}}{\left(q^{2} ; q\right)_{k-1}\left(q^{2} ; q\right)_{k-2}}
$$




$$
\begin{aligned}
& =\frac{q^{19}}{1-q^{2}}+\sum_{k=3}^{\infty} \frac{q^{k^{2}+5 k+5}}{\left(q^{2} ; q\right)_{k-1}\left(q^{2} ; q\right)_{k-3}}\left(1+\frac{q^{k-1}}{1-q^{k-1}}\right) \\
& =\frac{q^{19}}{1-q^{2}}+\sum_{k=3}^{\infty} \frac{q^{k^{2}+5 k+5}}{\left(q^{2} ; q\right)_{k-1}\left(q^{2} ; q\right)_{k-3}}+\sum_{k=3}^{\infty} \frac{q^{k^{2}+6 k+4}}{\left(q^{2} ; q\right)_{k-1}\left(q^{2} ; q\right)_{k-2}}
\end{aligned}
$$

We next transform the last term of (9.12) as given below.

$$
\begin{aligned}
& \sum_{k=2}^{\infty} \frac{q^{k^{2}+6 k+5}}{\left(q^{2} ; q\right)_{k-1}\left(q^{2} ; q\right)_{k-1}} \\
= & \sum_{k=2}^{\infty} \frac{q^{k^{2}+6 k+5}}{\left(q^{2} ; q\right)_{k-1}\left(q^{3} ; q\right)_{k-2}}\left(1+\frac{q^{2}}{1-q^{2}}\right) \\
= & \sum_{k=2}^{\infty} \frac{q^{k^{2}+6 k+5}}{\left(q^{2} ; q\right)_{k-1}\left(q^{3} ; q\right)_{k-2}}+\sum_{k=2}^{\infty} \frac{q^{k^{2}+6 k+7}}{\left(q^{2} ; q\right)_{k-1}\left(q^{2} ; q\right)_{k-1}} \\
= & \sum_{k=2}^{\infty} \frac{q^{k^{2}+6 k+5}}{\left(q^{2} ; q\right)_{k-1}\left(q^{3} ; q\right)_{k-2}}+\frac{q^{23}}{\left(1-q^{2}\right)^{2}}+\sum_{k=3}^{\infty} \frac{q^{k^{2}+6 k+7}}{\left(q^{2} ; q\right)_{k-1}\left(q^{2} ; q\right)_{k-3}\left(1-q^{k}\right)} \cdot \frac{1}{1-q^{k-1}} .
\end{aligned}
$$

Note that

$$
\frac{1}{1-q^{k-1}}=1+\frac{q^{k-1}\left(1-q^{4}\right)}{1-q^{k-1}}+\frac{q^{k+3}}{1-q^{k-1}} .
$$

Substituting (9.15) into (9.14) and with some simplification, we deduce that

$$
\begin{aligned}
& \sum_{k=2}^{\infty} \frac{q^{k^{2}+6 k+5}}{\left(q^{2} ; q\right)_{k-1}\left(q^{2} ; q\right)_{k-1}} \\
= & \frac{q^{23}}{\left(1-q^{2}\right)^{2}}+\sum_{k=2}^{\infty} \frac{q^{k^{2}+6 k+5}}{\left(q^{2} ; q\right)_{k-1}\left(q^{3} ; q\right)_{k-2}}+\sum_{k=3}^{\infty} \frac{q^{k^{2}+6 k+7}}{\left(q^{2} ; q\right)_{k-1}\left(q^{2} ; q\right)_{k-3}\left(1-q^{k}\right)} \\
& +\sum_{k=3}^{\infty} \frac{q^{k^{2}+7 k+6}\left(1+q^{2}\right)}{\left(q^{2} ; q\right)_{k-1}\left(q^{3} ; q\right)_{k-2}}+\sum_{k=3}^{\infty} \frac{q^{k^{2}+7 k+10}}{\left(q^{2} ; q\right)_{k-1}\left(q^{2} ; q\right)_{k-1}} .
\end{aligned}
$$

Substituting (9.13) and (9.16) into (9.12), we obtain (9.9). Thus the lemma has been verified.

We are now in a position to give a proof of Theorem 9.1 in light of Lemmas 9.2, 9.3 and 9.4 .

Proof of Theorem 9.1. Substituting (9.3), (9.6) and (9.9) into (9.1), we arrive at

$$
\sum_{n=0}^{\infty}(M(0, n)-M(1, n)) q^{n}
$$




$$
\begin{aligned}
= & -2 q+q^{3}+q^{4}-q^{7}-q^{9}+\frac{q^{10}}{1-q^{2}}-\frac{q^{11}}{1-q^{2}}+\frac{q^{12}}{1-q^{2}}-\frac{q^{19}}{1-q^{2}}-\frac{q^{23}}{\left(1-q^{2}\right)^{2}} \\
& +\left(\sum_{k=3}^{\infty} \frac{q^{k^{2}+4 k}}{\left(q^{2} ; q\right)_{k-2}\left(q^{3} ; q\right)_{k-3}}-\sum_{k=2}^{\infty} \frac{q^{k^{2}+6 k+5}}{\left(q^{2} ; q\right)_{k-1}\left(q^{3} ; q\right)_{k-2}}\right) \\
& +\left(\sum_{k=3}^{\infty} \frac{q^{k^{2}+4 k-1}}{\left(q^{2} ; q\right)_{k-2}\left(q^{2} ; q\right)_{k-3}}-\sum_{k=3}^{\infty} \frac{q^{k^{2}+6 k+4}}{\left(q^{2} ; q\right)_{k-1}\left(q^{2} ; q\right)_{k-2}}\right) \\
& +\left(\sum_{k=3}^{\infty} \frac{q^{k^{2}+3 k}}{\left(q^{2} ; q\right)_{k-3}\left(q^{2} ; q\right)_{k-3}}-\sum_{k=3}^{\infty} \frac{q^{k^{2}+7 k+10}}{\left(q^{2} ; q\right)_{k-1}\left(q^{2} ; q\right)_{k-1}}\right) \\
& +\left(\sum_{k=3}^{\infty} \frac{q^{k^{2}+5 k}}{\left(q^{2} ; q\right)_{k-1}\left(q^{3} ; q\right)_{k-2}}-\sum_{k=2}^{\infty} \frac{q^{k^{2}+5 k+3}}{\left(q^{2} ; q\right)_{k-1}\left(q^{3} ; q\right)_{k-2}}\right) \\
& +\left(\sum_{k=3}^{\infty} \frac{q^{k^{2}+5 k}}{\left(q^{2} ; q\right)_{k-1}\left(q^{2} ; q\right)_{k-3}}-\sum_{k=3}^{\infty} \frac{q^{k^{2}+5 k+5}}{\left(q^{2} ; q\right)_{k-2}\left(q^{2} ; q\right)_{k-3}}-\sum_{k=3}^{\infty} \frac{q^{k^{2}+4 k}}{\left(q^{2} ; q\right)_{k-1}\left(q^{2} ; q\right)_{k-3}}\right) \\
& +\left(\sum_{k=3}^{\infty} \frac{q^{k^{2}+5 k}}{\left.\left(q^{2} ; q\right)_{k-1}\left(q^{2} ; q\right)_{k-3}(1)-q^{k}\right)}\right)
\end{aligned}
$$

We proceed to simplify seven differences in the above identity. Note that

$$
\sum_{k=3}^{\infty} \frac{q^{k^{2}+4 k}}{\left(q^{2} ; q\right)_{k-2}\left(q^{3} ; q\right)_{k-3}}=\sum_{k=2}^{\infty} \frac{q^{k^{2}+6 k+5}}{\left(q^{2} ; q\right)_{k-1}\left(q^{3} ; q\right)_{k-2}}
$$

so (9.18) is equal to 0 .

We now consider the difference (9.19). Observe that

$$
\sum_{k=3}^{\infty} \frac{q^{k^{2}+4 k-1}}{\left(q^{2} ; q\right)_{k-2}\left(q^{2} ; q\right)_{k-3}}=\sum_{k=2}^{\infty} \frac{q^{k^{2}+6 k+4}}{\left(q^{2} ; q\right)_{k-1}\left(q^{2} ; q\right)_{k-2}}
$$

Hence (9.19) is equal to

$$
\sum_{k=2}^{\infty} \frac{q^{k^{2}+6 k+4}}{\left(q^{2} ; q\right)_{k-1}\left(q^{2} ; q\right)_{k-2}}-\sum_{k=3}^{\infty} \frac{q^{k^{2}+6 k+4}}{\left(q^{2} ; q\right)_{k-1}\left(q^{2} ; q\right)_{k-2}}=\frac{q^{20}}{1-q^{2}}
$$

Note that

$$
\sum_{k=3}^{\infty} \frac{q^{k^{2}+3 k}}{\left(q^{2} ; q\right)_{k-3}\left(q^{2} ; q\right)_{k-3}}=\sum_{k=1}^{\infty} \frac{q^{k^{2}+7 k+10}}{\left(q^{2} ; q\right)_{k-1}\left(q^{2} ; q\right)_{k-1}}
$$


so (9.20) is equal to

$$
\sum_{k=1}^{\infty} \frac{q^{k^{2}+7 k+10}}{\left(q^{2} ; q\right)_{k-1}\left(q^{2} ; q\right)_{k-1}}-\sum_{k=3}^{\infty} \frac{q^{k^{2}+7 k+10}}{\left(q^{2} ; q\right)_{k-1}\left(q^{2} ; q\right)_{k-1}}=q^{18}+\frac{q^{28}}{\left(1-q^{2}\right)^{2}}
$$

For (9.21), we see that

$$
\begin{aligned}
& \sum_{k=3}^{\infty} \frac{q^{k^{2}+5 k}}{\left(q^{2} ; q\right)_{k-1}\left(q^{3} ; q\right)_{k-2}}-\sum_{k=2}^{\infty} \frac{q^{k^{2}+5 k+3}}{\left(q^{2} ; q\right)_{k-1}\left(q^{3} ; q\right)_{k-2}} \\
= & \sum_{k=3}^{\infty} \frac{q^{k^{2}+5 k}}{\left(q^{2} ; q\right)_{k-1}\left(q^{3} ; q\right)_{k-2}}-\frac{q^{17}}{1-q^{2}}-\sum_{k=3}^{\infty} \frac{q^{k^{2}+5 k+3}}{\left(q^{2} ; q\right)_{k-1}\left(q^{3} ; q\right)_{k-2}} \\
= & -\frac{q^{17}}{1-q^{2}}+\sum_{k=3}^{\infty} \frac{q^{k^{2}+5 k}\left(1-q^{3}\right)}{\left(q^{2} ; q\right)_{k-1}\left(q^{3} ; q\right)_{k-2}} \\
= & -\frac{q^{17}}{1-q^{2}}+\sum_{k=3}^{\infty} \frac{q^{k^{2}+5 k}}{\left(q^{4} ; q\right)_{k-3}\left(q^{2} ; q\right)_{k-1}}
\end{aligned}
$$

The difference (9.22) can be simplified as follows:

$$
\begin{aligned}
& \sum_{k=3}^{\infty} \frac{q^{k^{2}+5 k}}{\left(q^{2} ; q\right)_{k-1}\left(q^{2} ; q\right)_{k-3}}-\sum_{k=3}^{\infty} \frac{q^{k^{2}+5 k+5}}{\left(q^{2} ; q\right)_{k-1}\left(q^{2} ; q\right)_{k-3}} \\
= & \sum_{k=3}^{\infty} \frac{q^{k^{2}+5 k}\left(1-q^{5}\right)}{\left(q^{2} ; q\right)_{k-1}\left(q^{2} ; q\right)_{k-3}} \\
= & \sum_{k=3}^{\infty} \frac{q^{k^{2}+5 k}\left(1-q^{2}+q^{2}-q^{5}\right)}{\left(q^{2} ; q\right)_{k-1}\left(q^{2} ; q\right)_{k-3}} \\
= & \sum_{k=3}^{\infty} \frac{q^{k^{2}+5 k}\left(1-q^{2}\right)}{\left(q^{2} ; q\right)_{k-1}\left(q^{2} ; q\right)_{k-3}}+\sum_{k=3}^{\infty} \frac{q^{k^{2}+5 k+2}\left(1-q^{3}\right)}{\left(q^{2} ; q\right)_{k-1}\left(q^{2} ; q\right)_{k-3}} \\
= & \sum_{k=3}^{\infty} \frac{q^{k^{2}+5 k}}{\left(q^{3} ; q\right)_{k-2}\left(q^{2} ; q\right)_{k-3}}+\sum_{k=3}^{\infty} \frac{q^{k^{2}+5 k+2}}{\left(1-q^{2}\right)\left(q^{4} ; q\right)_{k-3}\left(q^{2} ; q\right)_{k-3}} .
\end{aligned}
$$

For (9.23), we have

$$
\begin{aligned}
& \sum_{k=3}^{\infty} \frac{q^{k^{2}+4 k}}{\left(q^{2} ; q\right)_{k-2}\left(q^{2} ; q\right)_{k-3}}-\sum_{k=3}^{\infty} \frac{q^{k^{2}+6 k+7}}{\left(q^{2} ; q\right)_{k-1}\left(q^{2} ; q\right)_{k-3}\left(1-q^{k}\right)} \\
= & \sum_{k=2}^{\infty} \frac{q^{k^{2}+6 k+5}}{\left(q^{2} ; q\right)_{k-1}\left(q^{2} ; q\right)_{k-2}}-\sum_{k=3}^{\infty} \frac{q^{k^{2}+6 k+7}}{\left(q^{2} ; q\right)_{k-1}\left(q^{2} ; q\right)_{k-3}\left(1-q^{k}\right)}
\end{aligned}
$$




$$
\begin{aligned}
& =\frac{q^{21}}{1-q^{2}}+\sum_{k=3}^{\infty} \frac{q^{k^{2}+6 k+5}}{\left(q^{2} ; q\right)_{k-1}\left(q^{2} ; q\right)_{k-2}}-\sum_{k=3}^{\infty} \frac{q^{k^{2}+6 k+7}}{\left(q^{2} ; q\right)_{k-1}\left(q^{2} ; q\right)_{k-3}\left(1-q^{k}\right)} \\
& =\frac{q^{21}}{1-q^{2}}+\sum_{k=3}^{\infty} \frac{q^{k^{2}+6 k+5}}{\left(q^{2} ; q\right)_{k-1}\left(q^{2} ; q\right)_{k-3}}\left(\frac{1}{1-q^{k-1}}-\frac{q^{2}}{1-q^{k}}\right) \\
& =\frac{q^{21}}{1-q^{2}}+\sum_{k=3}^{\infty} \frac{q^{k^{2}+6 k+5}}{\left(q^{2} ; q\right)_{k-1}\left(q^{2} ; q\right)_{k-3}}\left(\sum_{i=0}^{\infty} q^{(k-1) i}-\sum_{i=0}^{\infty} q^{k i+2}\right) \\
& =\frac{q^{21}}{1-q^{2}}+\sum_{k=3}^{\infty} \sum_{i=0}^{\infty} \frac{q^{k^{2}+6 k+5+(k-1) i}\left(1-q^{i+2}\right)}{\left(q^{2} ; q\right)_{k-1}\left(q^{2} ; q\right)_{k-3}} .
\end{aligned}
$$

Finally, we transform (9.24) as given below:

$$
\begin{aligned}
& \sum_{k=3}^{\infty} \frac{q^{k^{2}+5 k}}{\left(q^{2} ; q\right)_{k-1}\left(q^{3} ; q\right)_{k-3}}-\sum_{k=3}^{\infty} \frac{q^{k^{2}+7 k+6}\left(1+q^{2}\right)}{\left(q^{2} ; q\right)_{k-1}\left(q^{3} ; q\right)_{k-2}} \\
= & \left(\frac{q^{24}}{\left(q^{2} ; q\right)_{2}}+\sum_{k=3}^{\infty} \frac{q^{k^{2}+7 k+6}}{\left(q^{2} ; q\right)_{k}\left(q^{3} ; q\right)_{k-2}}\right)-\sum_{k=3}^{\infty} \frac{q^{k^{2}+7 k+6}\left(1+q^{2}\right)}{\left(q^{2} ; q\right)_{k-1}\left(q^{3} ; q\right)_{k-2}} \\
= & \frac{q^{24}}{\left(q^{2} ; q\right)_{2}}+\sum_{k=3}^{\infty} \frac{q^{k^{2}+7 k+6}}{\left(q^{2} ; q\right)_{k-1}\left(q^{3} ; q\right)_{k-2}}\left(\frac{1}{1-q^{k+1}}-\left(1+q^{2}\right)\right) \\
= & \frac{q^{24}}{\left(q^{2} ; q\right)_{2}}+\sum_{k=3}^{\infty} \frac{q^{k^{2}+7 k+6}}{\left(q^{2} ; q\right)_{k-1}\left(q^{3} ; q\right)_{k-2}}\left(\frac{q^{2 k+2}}{1-q^{k+1}}-q^{2}\left(1-q^{k-1}\right)\right) \\
= & \frac{q^{24}}{\left(q^{2} ; q\right)_{2}}+\sum_{k=3}^{\infty} \frac{q^{k^{2}+9 k+8}}{\left(q^{2} ; q\right)_{k}\left(q^{3} ; q\right)_{k-2}}-\sum_{k=3}^{\infty} \frac{q^{k^{2}+7 k+8}}{\left(q^{2} ; q\right)_{k-3}\left(1-q^{k}\right)\left(q^{3} ; q\right)_{k-2}} \\
= & \frac{q^{24}}{\left(q^{2} ; q\right)_{2}}+\sum_{k=3}^{\infty} \frac{q^{k^{2}+9 k+8}}{\left(q^{2} ; q\right)_{k}\left(q^{3} ; q\right)_{k-2}}-\sum_{k=2}^{\infty} \frac{q^{k^{2}+9 k+16}}{\left(q^{2} ; q\right)_{k-2}\left(1-q^{k+1}\right)\left(q^{3} ; q\right)_{k-1}} \\
= & \frac{q^{24}}{\left(q^{2} ; q\right)_{2}}-\frac{q^{38}}{\left(1-q^{3}\right)^{2}}+\sum_{k=3}^{\infty} \frac{q^{k^{2}+9 k+8}}{\left(q^{2} ; q\right)_{k-2}\left(q^{3} ; q\right)_{k-2}\left(1-q^{k+1}\right)}\left(\frac{1}{1-q^{k}}-\frac{q^{8}}{1-q^{k+1}}\right) \\
= & \frac{q^{24}}{\left(q^{2} ; q\right)_{2}}-\frac{q^{38}}{\left(1-q^{3}\right)^{2}}+\sum_{k=3}^{\infty} \frac{q^{k^{2}+9 k+8}}{\left(q^{2} ; q\right)_{k-2}\left(q^{3} ; q\right)_{k-2}\left(1-q^{k+1}\right)}\left(\sum_{i=0}^{\infty} q^{i k}-\sum_{i=0}^{\infty} q^{i(k+1)+8}\right) \\
= & \frac{q^{24}}{\left(q^{2} ; q\right)_{2}}-\frac{q^{38}}{\left(1-q^{3}\right)^{2}}+\sum_{k=3}^{\infty} \sum_{i=0}^{\infty} \frac{q^{k^{2}+9 k+8+i k}\left(1-q^{i+8}\right)}{\left(q^{2} ; q\right)_{k-2}\left(q^{3} ; q\right)_{k-2}\left(1-q^{k+1}\right)} .
\end{aligned}
$$

Substituting (9.25) $\sim(9.30)$ into (9.17), we obtain (9.2). This completes the proof.

We are now ready to show that Theorem 1.7 holds when $m=1$ with the aid of the generating function of $M(0, n)-M(1, n)$ in Theorem 9.1 . 
Proof of Theorem 1.7 for $m=1$. Define

$$
\begin{aligned}
\sum_{n=0}^{\infty} T_{1}(n) q^{n}:= & -\frac{q^{11}}{1-q^{2}}-\frac{q^{17}}{1-q^{2}}-\frac{q^{19}}{1-q^{2}}-\frac{q^{23}}{\left(1-q^{2}\right)^{2}}-\frac{q^{38}}{\left(1-q^{3}\right)^{2}} \\
& +\sum_{k=3}^{\infty} \frac{q^{k^{2}+5 k}}{\left(q^{4} ; q\right)_{k-3}\left(q^{2} ; q\right)_{k-1}}+\sum_{k=3}^{\infty} \sum_{i=0}^{\infty} \frac{q^{k^{2}+6 k+5+(k-1) i}\left(1-q^{i+2}\right)}{\left(q^{2} ; q\right)_{k-1}\left(q^{2} ; q\right)_{k-3}} \\
& +\sum_{k=3}^{\infty} \sum_{i=0}^{\infty} \frac{q^{k^{2}+9 k+8+i k}\left(1-q^{i+8}\right)}{\left(q^{2} ; q\right)_{k-2}\left(q^{3} ; q\right)_{k-2}\left(1-q^{k+1}\right)}
\end{aligned}
$$

By Theorem 9.1, we see that when $n \geq 10$,

$$
M(0, n)-M(1, n) \geq T_{1}(n)
$$

To prove Theorem 1.7 for $m=1$, it suffices to establish the nonnegativity on $T_{1}(n)$.

By Lemma 3.5, we see that

$$
\sum_{k=3}^{\infty} \sum_{i=0}^{\infty} \frac{q^{k^{2}+6 k+5+(k-1) i}\left(1-q^{i+2}\right)}{\left(q^{2} ; q\right)_{k-1}\left(q^{2} ; q\right)_{k-3}}=\sum_{k=3}^{\infty} \sum_{i=0}^{\infty} \frac{q^{k^{2}+6 k+5+(k-1) i}}{\left(q^{4} ; q\right)_{k-3}\left(q^{2} ; q\right)_{k-3}} \cdot \frac{1-q^{i+2}}{\left(1-q^{2}\right)\left(1-q^{3}\right)}
$$

and

$$
\begin{aligned}
& \sum_{k=3}^{\infty} \sum_{i=0}^{\infty} \frac{q^{k^{2}+9 k+8+i k}\left(1-q^{i+8}\right)}{\left(q^{2} ; q\right)_{k-2}\left(q^{3} ; q\right)_{k-2}\left(1-q^{k+1}\right)} \\
& =\sum_{k=3}^{\infty} \sum_{i=0}^{\infty} \frac{q^{k^{2}+9 k+8+i k}}{\left(q^{3} ; q\right)_{k-3}\left(q^{4} ; q\right)_{k-3}\left(1-q^{k+1}\right)} \cdot \frac{1-q^{i+8}}{\left(1-q^{2}\right)\left(1-q^{3}\right)}
\end{aligned}
$$

have nonnegative power series coefficients. Define

$$
\sum_{n=0}^{\infty} H(n) q^{n}:=\frac{q^{36}}{\left(q^{2} ; q\right)_{3}}-\frac{q^{11}}{1-q^{2}}-\frac{q^{17}}{1-q^{2}}-\frac{q^{19}}{1-q^{2}}-\frac{q^{23}}{\left(1-q^{2}\right)^{2}}-\frac{q^{38}}{\left(1-q^{3}\right)^{2}}
$$

Note that

$$
\begin{aligned}
& \sum_{k=3}^{\infty} \frac{q^{k^{2}+5 k}}{\left(q^{4} ; q\right)_{k-3}\left(q^{2} ; q\right)_{k-1}}-\frac{q^{36}}{\left(q^{2} ; q\right)_{3}} \\
& =\frac{q^{24}}{\left(q^{2} ; q\right)_{2}}+\frac{q^{36}}{\left(1-q^{4}\right)\left(q^{2} ; q\right)_{3}}-\frac{q^{36}}{\left(q^{2} ; q\right)_{3}}+\sum_{k=5}^{\infty} \frac{q^{k^{2}+5 k}}{\left(q^{4} ; q\right)_{k-3}\left(q^{2} ; q\right)_{k-1}} \\
& =\frac{q^{24}}{\left(q^{2} ; q\right)_{2}}+\frac{q^{40}}{\left(1-q^{4}\right)\left(q^{2} ; q\right)_{3}}+\sum_{k=5}^{\infty} \frac{q^{k^{2}+5 k}}{\left(q^{4} ; q\right)_{k-3}\left(q^{2} ; q\right)_{k-1}},
\end{aligned}
$$


which has nonnegative power series coefficients. Hence we derive that for $n \geq 11$,

$$
T_{1}(n) \geq H(n)
$$

We proceed to show that $H(n) \geq 0$ when $n \geq 106$. By (1.11), we see that

$$
\frac{q^{36}}{\left(q^{2} ; q\right)_{3}}=\sum_{n=36}^{\infty} p_{4}(n-36) q^{n}
$$

From (9.33), we find that for $n \geq 38$,

$$
H(n)= \begin{cases}p_{4}(n-36), & \text { if } n \equiv 0,4 \quad(\bmod 6), \\ p_{4}(n-36)-\frac{n-35}{3}, & \text { if } n \equiv 2 \quad(\bmod 6), \\ p_{4}(n-36)-\frac{n-21}{2}-3, & \text { if } n \equiv 1,3 \quad(\bmod 6), \\ p_{4}(n-36)-\frac{n-21}{2}-3-\frac{n-35}{3}, & \text { if } n \equiv 5 \quad(\bmod 6) .\end{cases}
$$

By Lemma 3.2 (3), we see that for $n \geq 48$,

$$
p_{4}(n-36) \geq 3\left(\frac{n-36}{12}-1\right)^{2}=\frac{n^{2}}{48}-2 n+48 .
$$

Hence we deduce that for $n \geq 106$,

$$
H(n) \geq p_{4}(n-36)-\frac{n-21}{2}-3-\frac{n-35}{3} \geq \frac{n^{2}}{48}-2 n+48-\frac{n-21}{2}-3-\frac{n-35}{3} \geq 0 .
$$

This implies that $T_{1}(n) \geq 0$ for $n \geq 106$, and so $M(0, n)-M(1, n) \geq 0$ when $n \geq 106$.

It can be checked that $M(0, n) \geq M(1, n)$ when $44 \leq n \leq 105$. Thus, we complete the proof of the theorem.

\section{Proofs of Theorem 1.9 and Conjecture 1.4}

In this section, we first prove Theorem 1.9, and then give a proof of Conjecture 1.4 with the aid of Theorem 1.7 and Theorem 1.9.

To prove Theorem 1.9, setting $m=0$ in Theorem 2.1, we see that

$$
\sum_{n=0}^{\infty} 21 M(0, n) q^{n}=21-21 q+\sum_{k=1}^{\infty} \frac{21 q^{k^{2}+2 k}}{(q ; q)_{k}\left(q^{2} ; q\right)_{k-1}} .
$$

From [2, Corollary 2.6],

$$
\sum_{n=0}^{\infty} p(n) q^{n}=1+\sum_{k=1}^{\infty} \frac{q^{k^{2}}}{(q ; q)_{k}^{2}}
$$


Subtracting (10.1) from (10.2), we obtain the following generating function:

$$
\sum_{n=0}^{\infty}(p(n)-21 M(0, n)) q^{n}=-20+21 q+\sum_{k=1}^{\infty} q^{k^{2}}\left(\frac{1}{(q ; q)_{k}^{2}}-\frac{21 q^{2 k}}{(q ; q)_{k}\left(q^{2} ; q\right)_{k-1}}\right) .
$$

For $k \geq 1$, define

$$
\sum_{n=0}^{\infty} g_{k}(n) q^{n}:=\frac{1}{(q ; q)_{k}^{2}}
$$

and

$$
\sum_{n=0}^{\infty} h_{k}(n) q^{n}:=\frac{q^{2 k}}{(q ; q)_{k}\left(q^{2} ; q\right)_{k-1}}
$$

This leads to for $n \geq 2$,

$$
p(n)-21 M(0, n)=\sum_{k=1}^{\infty}\left(g_{k}\left(n-k^{2}\right)-21 h_{k}\left(n-k^{2}\right)\right) .
$$

The following theorem establishes the nonnegativity of $g_{k}(n)-21 h_{k}(n)$ which implies that $p(n) \geq 21 M(0, n)$ for $n \geq 76$. Furthermore, it is not difficult to check that $p(n) \geq$ $21 M(0, n)$ for $39 \leq n \leq 75$. Hence by (10.5), we see that Theorem 1.9 immediately follows from the following theorem.

Theorem 10.1. (1) $g_{1}(n) \geq 21 h_{1}(n)$ for $n \geq 20$.

(2) $g_{2}(n) \geq 21 h_{2}(n)$ for $n \geq 51$.

(3) $g_{3}(n) \geq 21 h_{3}(n)$ for $n \geq 67$.

(4) When $k \geq 4, g_{k}(n) \geq 21 h_{k}(n)$ for $n \geq 0$.

Before proving Theorem [10.1, we first derive the following recurrences of $g_{k}(n)$ and $h_{k}(n)$.

Lemma 10.2. For $k \geq 1$,

$$
g_{k}(n)=\sum_{i=0}^{\left\lfloor\frac{n}{k}\right\rfloor}(i+1) g_{k-1}(n-k i)
$$

and for $k \geq 2$,

$$
h_{k}(n)=\sum_{i=0}^{\left\lfloor\frac{n}{k}\right\rfloor-2}(i+1) h_{k-1}(n-k i-2)
$$


Proof. From the definition (10.3) of $g_{k}(n)$, we see that when $k \geq 1$,

$$
\begin{aligned}
\sum_{n=0}^{\infty} g_{k}(n) q^{n} & =\frac{1}{(q ; q)_{k}^{2}} \\
& =\frac{1}{(q ; q)_{k-1}^{2}} \cdot \frac{1}{\left(1-q^{k}\right)^{2}} \\
& =\sum_{n=0}^{\infty} g_{k-1}(n) q^{n} \cdot \sum_{i=0}^{\infty}(i+1) q^{k i} \\
& =\sum_{n=0}^{\infty} \sum_{i=0}^{\left\lfloor\frac{n}{k}\right\rfloor}(i+1) g_{k-1}(n-k i) q^{n}
\end{aligned}
$$

So we obtain the recurrence (10.6) by equating coefficients of $q^{n}$ on both sides of the above identity.

Proceeding as in the proof of (10.6), we have

$$
h_{k}(n)=\sum_{i=0}^{\infty}(i+1) h_{k-1}(n-k i-2) .
$$

From (10.4), we see that $h_{k}(n)=0$ if $n<2 k$. Thus (10.8) can be written as follows:

$$
h_{k}(n)=\sum_{i=0}^{\left\lfloor\frac{n}{k}\right\rfloor-2}(i+1) h_{k-1}(n-k i-2)
$$

which is (10.7). This completes the proof.

In order to prove Theorem 10.1, we also require the following lemma.

Lemma 10.3. When $k \geq 1$ and $n \geq 0$,

$$
g_{k}(n+1) \geq g_{k}(n)
$$

and

$$
h_{k}(n+1) \geq h_{k}(n) .
$$

Furthermore, when $k \geq 2$ and $n \geq 0$,

$$
k^{2} h_{k}(n) \leq n^{2} h_{k-1}(n) .
$$

Proof. By definition, it is clear that for $k \geq 1$,

$$
1+\sum_{n=1}^{\infty}\left(g_{k}(n)-g_{k}(n-1)\right) q^{n}=\frac{1-q}{(q ; q)_{k}^{2}}=\frac{1}{(q ; q)_{k}\left(q^{2} ; q\right)_{k-1}}
$$


which obviously has nonnegative power series coefficients. This yields (10.9).

Similarly, by (10.4), we see that for $k \geq 1$,

$$
\sum_{n=1}^{\infty}\left(h_{k}(n)-h_{k}(n-1)\right) q^{n}=\frac{(1-q) q^{2 k}}{(q ; q)_{k}\left(q^{2} ; q\right)_{k-1}}=\frac{q^{2 k}}{\left(q^{2} ; q\right)_{k-1}^{2}},
$$

which also has nonnegative power series coefficients. Hence (10.10) is valid.

We next prove (10.11). By (10.7), we see that when $k \geq 2$,

$$
k^{2} h_{k}(n)=k^{2} \sum_{i=0}^{\left\lfloor\frac{n}{k}\right\rfloor-2}(i+1) h_{k-1}(n-k i-2) .
$$

From (10.10), we find that when $k \geq 2$ and $n \geq 0$,

$$
\begin{aligned}
k^{2} h_{k}(n) & \leq k^{2} \sum_{i=0}^{\left\lfloor\frac{n}{k}\right\rfloor-2}(i+1) h_{k-1}(n) \\
& \leq k^{2}\left(\left\lfloor\frac{n}{k}\right\rfloor-1\right)^{2} h_{k-1}(n) \\
& \leq n^{2} h_{k-1}(n),
\end{aligned}
$$

as desired. This completes the proof.

We are now in a position to prove Theorem 10.1.

Proof of Theorem 10.1. (1) When $k=1$, it follows immediately from (10.6) that $g_{1}(n)=$ $n+1$ by noting that $g_{0}(0)=1$ and $g_{0}(n)=0$ for $n \geq 1$. On the other hand, by the definition of $h_{k}(n)$, we see that $h_{1}(0)=h_{1}(1)=0$, and $h_{1}(n)=1$ for $n \geq 2$. Hence $g_{1}(n) \geq 21 h_{1}(n)$ when $n \geq 20$.

(2) When $k=2$, we first claim that when $n \geq 0$,

$$
g_{2}(n) \geq \frac{n^{3}}{24}
$$

Set $n=2 t+j$, where $t \geq 0$ and $j=0$ or 1 . Notice that $g_{1}(n)=n+1$, by (10.6), we see that for $n \geq 0$,

$$
\begin{aligned}
g_{2}(n) & =\sum_{i=0}^{\lfloor n / 2\rfloor}(i+1) g_{1}(n-2 i) \\
& =\sum_{i=0}^{\lfloor n / 2\rfloor}(i+1)(n-2 i+1)
\end{aligned}
$$




$$
\begin{aligned}
& \geq \sum_{i=0}^{t}(i+1)(2 t-2 i+1) \\
& =\frac{t^{3}}{3}+\frac{3 t^{2}}{2}+\frac{13 t}{6}+1
\end{aligned}
$$

Hence, we derive that

$$
g_{2}(n) \geq \frac{t^{3}}{3}+\frac{3 t^{2}}{2}+\frac{13 t}{6}+1 \geq \frac{(t+1)^{3}}{3} .
$$

Since $n \leq 2 t+2$, we deduce from (10.13) that for $n \geq 0$,

$$
g_{2}(n) \geq \frac{(t+1)^{3}}{3} \geq \frac{n^{3}}{24} .
$$

This yields (10.12).

On the other hand, since $h_{1}(n)=0$ or 1 for $n \geq 0$, and by (10.11), we find that for $n \geq 0$,

$$
h_{2}(n) \leq \frac{n^{2}}{4} .
$$

Together with (10.12), we derive that for $n \geq 126$,

$$
g_{2}(n) \geq \frac{n^{3}}{24} \geq \frac{21 n^{2}}{4} \geq 21 h_{2}(n)
$$

Moreover, it can be checked that $g_{2}(n) \geq 21 h_{2}(n)$ when $51 \leq n \leq 125$. Hence we conclude that $g_{2}(n) \geq 21 h_{2}(n)$ for $n \geq 51$.

(3) By suitable modification to the proof of (10.12), we can show that

$$
g_{3}(n) \geq \frac{n^{5}}{4320}
$$

On the other hand, combining (10.11) and (10.14), we find that when $n \geq 0$,

$$
h_{3}(n) \leq \frac{n^{4}}{36}
$$

Hence by (10.15) and (10.16), we derive that when $n \geq 2520$,

$$
g_{3}(n) \geq \frac{n^{5}}{4320} \geq \frac{21 n^{4}}{36} \geq 21 h_{3}(n) .
$$

Furthermore, it is easy to check that $g_{3}(n) \geq 21 h_{3}(n)$ when $67 \leq n \leq 2519$. Hence we conclude that $g_{3}(n) \geq 21 h_{3}(n)$ when $n \geq 67$.

(4) For $k \geq 4$, we will prove that $g_{k}(n) \geq 21 h_{k}(n)$ when $n \geq 0$ by induction on $k$. 
When $k=4$, using the same method as above and after some tedious but straightforward calculation, we deduce that when $n \geq 8$,

$$
g_{4}(n) \geq \frac{1}{2903040} n^{7}
$$

Here we omit the detail.

On the other hand, from (10.11) and (10.16), we deduce that when $n \geq 0$,

$$
h_{4}(n) \leq \frac{n^{6}}{576}
$$

Hence, when $n \geq 105840$,

$$
g_{4}(n) \geq \frac{n^{7}}{2903040} \geq \frac{21 n^{6}}{576} \geq 21 h_{4}(n) .
$$

Furthermore, it can be checked that $g_{4}(n) \geq 21 h_{4}(n)$ when $0 \leq n \leq 105839$, so $g_{4}(n) \geq$ $21 h_{4}(n)$ for $n \geq 0$.

We now assume that there exists $k \geq 5$ such that $g_{k-1}(n) \geq 21 h_{k-1}(n)$ for $n \geq 0$. We aim to show that for $n \geq 0$,

$$
g_{k}(n) \geq 21 h_{k}(n) .
$$

From (10.6) and (10.9), we derive that

$$
\begin{aligned}
g_{k}(n) & =\sum_{i=0}^{\left\lfloor\frac{n}{k}\right\rfloor}(i+1) g_{k-1}(n-k i) \\
& \geq \sum_{i=0}^{\left\lfloor\frac{n}{k}\right\rfloor}(i+1) g_{k-1}(n-k i-2) .
\end{aligned}
$$

By the induction hypothesis, we have

$$
g_{k-1}(n-k i-2) \geq 21 h_{k-1}(n-k i-2) .
$$

Hence

$$
g_{k}(n) \geq 21 \sum_{i=0}^{\left\lfloor\frac{n}{k}\right\rfloor}(i+1) h_{k-1}(n-k i-2)
$$

From (10.7), we have

$$
h_{k}(n)=\sum_{i=0}^{\left\lfloor\frac{n}{k}\right\rfloor-2}(i+1) h_{k-1}(n-k i-2) .
$$

It follows that for $n \geq 0$,

$$
g_{k}(n) \geq 21 h_{k}(n) .
$$

This completes the proof. 
We conclude this section with a proof of Conjecture 1.4. Let $N(\leq m, n)$ denote the number of partitions of $n$ with rank less than or equal to $m$, and let $M(\leq m, n)$ denote the number of partitions of $n$ with crank less than or equal to $m$. Bringmann and Mahlburg [14] conjectured that for $n \geq 1$ and $m \leq 0$,

$$
M(\leq m, n) \leq N(\leq m+1, n),
$$

which has been proved by Chen, Ji and Zang in [19].

By using the following two symmetries of ranks and cranks (see [22, 23]):

$$
M(m, n)=M(-m, n),
$$

and

$$
N(m, n)=N(-m, n),
$$

it is not difficult to derive from (10.17) that for $n \geq 1$ and $m \geq 0$,

$$
N(\leq m-1, n) \leq M(\leq m, n) .
$$

We are now in a position to give a proof of Conjecture 1.4 by means of Theorem 1.7 and Theorem 1.9 as well as (1.5), (10.17), (10.18) and (10.20).

Proof of Conjecture 1.4. Setting $m=-2$ in (10.17), we see that for $n \geq 1$,

$$
M(\leq-2, n) \leq N(\leq-1, n) .
$$

Setting $m=2$ in (10.20), we see that for $n \geq 1$,

$$
M(\leq 2, n) \geq N(\leq 1, n) .
$$

Subtracting (10.21) from (10.22) leads to

$$
N(0, n)+N(1, n) \leq M(-1, n)+M(0, n)+M(1, n)+M(2, n),
$$

for $n \geq 1$. By (10.18), we see that for $n \geq 1$,

$$
M(-1, n)=M(1, n)
$$

and so (10.23) becomes

$$
N(0, n)+N(1, n) \leq M(0, n)+2 M(1, n)+M(2, n) .
$$

By Theorem 1.7, we see that for $n \geq 44$,

$$
M(0, n) \geq M(1, n) \geq M(2, n),
$$

and so by (10.24), we derive that for $n \geq 44$,

$$
N(0, n)+N(1, n) \leq 4 M(0, n) .
$$


From (1.5), we see that for $n \geq 7$,

$$
\operatorname{ospt}(n)<\frac{p(n)}{4}+\frac{N(0, n)}{2}-\frac{M(0, n)}{4}+\frac{N(1, n)}{2} \text {. }
$$

Applying (10.25) in (10.26), we are led to

$$
\operatorname{ospt}(n)<\frac{p(n)}{4}+\frac{7 M(0, n)}{4}
$$

for $n \geq 44$. Appealing to Theorem [1.9, we see that for $n \geq 39$,

$$
21 M(0, n) \leq p(n)
$$

Hence we arrive at

$$
\operatorname{ospt}(n)<\frac{p(n)}{3}
$$

for $n \geq 44$. Furthermore, it is easy to check that $\operatorname{ospt}(n)<\frac{p(n)}{3}$ when $10 \leq n \leq 43$. Thus, we complete the proof of Conjecture 1.4.

\section{Conjectures}

Recall that a sequence $\left\{a_{i}\right\}_{1 \leq i \leq n}$ is called log-concave if for $2 \leq i \leq n-1, a_{i}$ satisfies the following inequality:

$$
a_{i}^{2} \geq a_{i-1} a_{i+1}
$$

It is well known that if a sequence $\left\{a_{i}\right\}$ of positive integers is log-concave, then $\left\{a_{i}\right\}$ is unimodal, see [32, P.124, Ex.50].

An interesting phenomenon occurs when we consider the log-concavity of $M(m, n)$. In particular, for $72 \leq n \leq 10000$ and $72-n \leq m \leq n-72$ (tested with Mathematica), the following inequality holds,

$$
M(m, n)^{2} \geq M(m-1, n) M(m+1, n) .
$$

In this case, we would like to make the following conjecture.

Conjecture 11.1. For $n \geq 72$ and $72-n \leq m \leq n-72$,

$$
M(m, n)^{2} \geq M(m-1, n) M(m+1, n) .
$$

In other words, for $n \geq 72$, the sequence $\{M(m, n)\}_{|m| \leq n-71}$ is log-concave. 
Obviously, this conjecture implies the sequence $\{M(m, n)\}_{|m| \leq n-71}$ is unimodal when $n \geq 72$. More precisely, when $n \geq 72$ and $1 \leq m \leq n-71$,

$$
M(m-1, n) \geq M(m, n) .
$$

It should be noted that the inequality (11.2) is also valid when $n \geq 72$ and $n-70 \leq m \leq$ $n-1$, which follows immediately from the following lemma. Hence if Conjecture 11.1 is proved to be true, then we could derive that the sequence $\{M(m, n)\}_{|m| \leq n-1}$ is unimodal when $n \geq 44$ which is Corollary 1.8 .

Lemma 11.2. For $n \geq 2 i \geq 4$,

$$
M(n-i, n)=p_{i}(i)
$$

where $p_{r}(n)$ counts the number of partitions of $n$ with parts taken from $\{2,3, \ldots, r\}$, as defined in (1.11).

Proof. Let $\lambda=\left(\lambda_{1}, \lambda_{2}, \ldots, \lambda_{\ell}\right)$ be a partition of $n$ counted by $M(n-i, n)$. Let $n_{1}(\lambda)$ denote the number of 1 's in $\lambda$. We claim that $n_{1}(\lambda)=0$ when $n \geq 2 i$. Otherwise, by the definition of crank, we see that there are exactly $n-i+n_{1}(\lambda)$ parts in $\lambda$ strictly larger than $n_{1}(\lambda)$. Note that $n_{1}(\lambda) \geq 1$, so there are at least $n-i+n_{1}(\lambda)$ parts in $\lambda$ not less than 2. This leads to

$$
n=\sum_{i=1}^{\ell} \lambda_{i} \geq 2\left(n-i+n_{1}(\lambda)\right) \geq 2 n-2 i+2 \geq n+2,
$$

a contradiction. Hence $n_{1}(\lambda)=0$ and by the definition of crank, we see that $\lambda_{1}=n-i$, which implies

$$
\sum_{i=2}^{\ell} \lambda_{i}=n-\lambda_{1}=i
$$

Moreover, $n_{1}(\lambda)=0$ implies that $\lambda_{\ell} \geq 2$. Hence $\left(\lambda_{2}, \ldots, \lambda_{\ell}\right)$ is counted by $p_{i}(i)$. Conversely, let $\mu=\left(\mu_{1}, \ldots, \mu_{j}\right)$ be a partition counted by $p_{i}(i)$, notice that $n-i \geq i \geq \mu_{1}$, so the partition $\left(n-i, \mu_{1}, \ldots, \mu_{j}\right)$ is counted by $M(n-i, n)$. Hence the equality (11.3) holds.

We proceed to illustrate the inequality (11.2) is true when $n \geq 72$ and $n-70 \leq m \leq$ $n-1$ with the aid of Lemma 11.2. By a direct calculation, it is easy to check that when $2 \leq i \leq 70$,

$$
p_{i+1}(i+1) \geq p_{i}(i) .
$$

Thus from Lemma 11.2, we see that when $n \geq 142$ and $2 \leq i \leq 70$,

$$
M(n-i-1, n) \geq M(n-i, n) .
$$


This leads to $M(m-1, n) \geq M(m, n)$ for $n \geq 142$ and $n-70 \leq m \leq n-2$. Note that $M(n-2, n)=1>0=M(n-1, n)$. Hence $M(m-1, n) \geq M(m, n)$ is valid for $n \geq 142$ and $n-70 \leq m \leq n-1$. After checking the small cases for $72 \leq n \leq 141$, we have verified $M(m-1, n) \geq M(m, n)$ for $n \geq 72$ and $n-70 \leq m \leq n-1$.

The similar phenomenon also occurs for $N(m, n)$. We have the following conjecture.

Conjecture 11.3. For $n \geq 73$ and $73-n \leq m \leq n-73$,

$$
N(m, n)^{2} \geq N(m-1, n) N(m+1, n) .
$$

In other words, for $n \geq 73$, the sequence $\{N(m, n)\}_{|m| \leq n-72}$ is log-concave.

In [17], Chan and Mao raised a problem of finding the condition such that the inequality (11.5) holds. By the calculation with Mathematica, we have the following conjecture.

Conjecture 11.4. For $n \geq 39$ and $1 \leq m \leq n-2$,

$$
N(m-1, n) \geq N(m, n) .
$$

By the symmetry $N(m, n)=N(-m, n)$, we see that Conjecture 11.4 implies the sequence $\{N(m, n)\}_{|m| \leq n-2}$ is unimodal for $n \geq 39$.

It is clear that Conjecture 11.3 implies the inequality (11.5) holds when $n \geq 73$ and $1 \leq m \leq n-72$. We will prove that the inequality (11.5) also holds when $n \geq 73$ and $n-71 \leq m \leq n-2$ by using the following lemma. Therefore, the inequality (11.5) holds when $n \geq 73$ and $1 \leq m \leq n-2$. Furthermore, it is easy to check that (11.5) holds when $39 \leq n \leq 72$ and $1 \leq m \leq n-2$. Hence we could say that Conjecture 11.3 implies Conjecture 11.4

Lemma 11.5. For $n \geq 2 i \geq 4$,

$$
N(n-i, n)=\sum_{\ell=2}^{\lfloor(i+1) / 2\rfloor} p(i-2 \ell+1, \ell-1) .
$$

where $p(n, r)$ denotes the number of partitions of $n$ with at most $r$ parts, as defined in (3.5).

Proof. Let $\lambda=\left(\lambda_{1}, \ldots, \lambda_{\ell}\right)$ be a partition counted by $N(n-i, n)$. We claim that $\ell \geq 2$. Otherwise if $\ell=1$, then $\lambda=(n)$ which is counted by $N(n-1, n)$, which contradicts to the fact that $\lambda$ is counted by $N(n-i, n)$ where $i \geq 2$. By the definition of rank, we see that $\lambda_{1}=n-i+\ell$, so

$$
\sum_{j=2}^{\ell}\left(\lambda_{j}-1\right)=n-\lambda_{1}-\ell+1=i-2 \ell+1 \geq 0
$$


Hence $\mu=\left(\lambda_{2}-1, \lambda_{3}-1, \ldots, \lambda_{\ell}-1\right)$ is a partition counted by $p(i-2 \ell+1, \ell-1)$. Furthermore, $\ell \leq(i+1) / 2$.

Conversely, for $i \geq 2$ and $2 \leq \ell \leq\lfloor(i+1) / 2\rfloor$, and let $\mu=\left(\mu_{1}, \mu_{2}, \ldots, \mu_{\ell-1}\right)$ be a partition counted by $p(i-2 \ell+1, \ell-1)$, where $\mu_{\ell-1} \geq 0$. Note that $n \geq 2 i$, so $\mu_{1} \leq i-2 \ell+1 \leq n-i+\ell-1$. Hence $\lambda=\left(n-i+\ell, \mu_{1}+1, \mu_{2}+1, \ldots, \mu_{\ell-1}+1\right)$ is a partition counted by $N(n-i, n)$. Thus we arrive at (11.6).

We proceed to show that the inequality (11.5) holds when $n \geq 73$ and $n-71 \leq m \leq$ $n-2$ with the aid of Lemma 11.5, By a straightforward calculation, it is easy to check that when $2 \leq i \leq 71$,

$$
\sum_{\ell=2}^{\lfloor(i+2) / 2\rfloor} p(i-2 \ell+2, \ell-1) \geq \sum_{\ell=2}^{\lfloor(i+1) / 2\rfloor} p(i-2 \ell+1, \ell-1) .
$$

Thus from Lemma 11.5, we see that when $n \geq 144$ and $2 \leq i \leq 71$,

$$
N(n-i-1, n) \geq N(n-i, n) .
$$

This leads to $N(m-1, n) \geq N(m, n)$ for $n \geq 144$ and $n-71 \leq m \leq n-2$. It is easy to check that $N(m-1, n) \geq N(m, n)$ for $73 \leq n \leq 143$ and $n-71 \leq m \leq n-2$. Hence $N(m-1, n) \geq N(m, n)$ when $n \geq 73$ and $n-71 \leq m \leq n-2$.

It should be noted that Conjecture 11.1 and Conjecture 11.3 have also been raised by Bringmann, Jennings-Shaffer and Mahlburg [13, Conjecture 4.3].

Acknowledgments. This work was supported by the National Science Foundation of China. We are greatly indebted to referees for their helpful suggestions that improved the presentation of this paper.

\section{References}

[1] S. Ahlgren and K. Ono, Congruence properties for the partition function, Proc. Natl. Acad. Sci. USA. 98 (23) (2001) 12882-12884.

[2] G.E. Andrews, The Theory of Partitions, Encyclopedia of Mathematics and its Applications, Vol. 2. Addison-Wesley Publishing Co., Reading, Mass.-London-Amsterdam, 1976.

[3] G.E. Andrews, Partitions: at the interface of $q$-series and modular forms, Ramanujan J. 7 (2003) 384-400.

[4] G.E. Andrews, The number of smallest parts in the partitions of $n$, J. Reine Angew. Math. 624 (2008) 133-142.

[5] G.E. Andrews, S.H. Chan and B. Kim, The odd moments of ranks and cranks, J. Combin. Theory Ser. A 120 (1) (2013) 77-91.

[6] G.E. Andrews, F.J. Dyson and R.C. Rhoades, On the distribution of the spt-crank, Mathematics 1 (2013) 76-88. 
[7] G.E. Andrews and F.G. Garvan, Dyson's crank of a partition, Bull. Amer. Math. Soc. 18 (2) (1988) 167-171.

[8] G.E. Andrews and F.G. Garvan, Ramanujan's "lost" notebook VI: The mock theta conjectures, Adv. Math. 73 (2) (1989) 242-255.

[9] G.E. Andrews, F.G. Garvan and J.L. Liang, Combinatorial interpretations of congruences for the spt-function, Ramanujan J. 29 (2012) 321-338.

[10] G.E. Andrews and K. Ono, Ramanujan's congruences and Dyson's crank, Proc. Natl. Acad. Sci. USA 102 (43) (2005) 15277.

[11] A.O.L. Atkin and P. Swinnerton-Dyer, Some properties of partitions, Proc. London Math. Soc. (3) 4 (1954) 84-106.

[12] K. Bringmann and J. Dousse, On Dyson's crank conjecture and the uniform asymptotic behavior of certain inverse theta functions, Trans. Amer. Math. Soc. 368 (5) (2016) 31413155.

[13] K. Bringmann, C. Jennings-Shaffer and K. Mahlburg, The asymptotic distribution of the rank for unimodal sequences, J. Number Theory (2021), https://doi.org/10.1016/j.jnt.2020.11.016

[14] K. Bringmann and K. Mahlburg, Inequalities between ranks and cranks, Proc. Amer. Math. Soc. 137 (8) (2009) 2567-2574.

[15] K. Bringmann and K. Ono, The $f(q)$ mock theta function conjecture and partition ranks, Invent. Math. 165 (2) (2006) 243-266.

[16] K. Bringmann and K. Ono, Dyson's ranks and Maass forms, Ann. Math. (2) 171 (1) (2010) 419-449.

[17] S.H. Chan and R. Mao, Inequalities for ranks of partitions and the first moment of ranks and cranks of partitions, Adv. Math. 258 (2014) 414-437.

[18] W.Y.C. Chen, K.Q. Ji and W.J.T. Zang, Proof of the Andrews-Dyson-Rhoades conjecture on the spt-crank, Adv. Math. 270 (2015) 60-96.

[19] W.Y.C. Chen, K.Q. Ji and W.J.T. Zang, Nearly equal distributions of the rank and the crank of partitions, Analytic number theory, modular forms and q-hypergeometric series, 157-177, Springer Proc. Math. Stat., 221, Springer, 2017.

[20] A. DeMorgan, On a new form of difference equation, Cambridge Math. J. 4 (1843) 87-90.

[21] F.J. Dyson, Some guesses in the theorey of partitions, Eureka (Cambridge) 8 (1944) 10-15.

[22] F.J. Dyson, A new symmetry of partitions, J. Combin. Theory Ser. A 7 (1969) 56-61.

[23] F.J. Dyson, Mappings and symmetries of partitions, J. Combin. Theory Ser. A 51 (2) (1989) 169-180.

[24] F.G. Garvan, New combinatorial interpretations of Ramanujan's partition congruences mod 5, 7 and 11, Trans. Amer. Math. Soc. 305 (1988) 47-77.

[25] F.G. Garvan, Combinatorial interpretations of Ramanujan's partition congruences, Ramanujan revisited, 29-45, Academic Press, Boston, MA, 1988.

[26] F.G. Garvan, The crank of partitions mod 8, 9 and 10, Trans. Amer. Math. Soc. 322 (1) (1990) 79-94.

[27] K. Glösel, Über die Zerlegung der ganzen Zahlen, Monatschefte Math. Phys. 7 (1896) 133-141. 
[28] B. Kim, E. Kim and J. Seo, Asymptotics for q-expansions involving partial theta functions, Discrete Math. 338 (2) (2015) 180-189.

[29] R. Lewis, On the ranks of partitions modulo 9, Bull. London Math. Soc. 23 (5) (1991) 417-421.

[30] K. Mahlburg, Partition congruences and the Andrews-Garvan-Dyson crank, Proc. Natl. Acad. Sci. USA. 102 (43) (2005) 15373-15376.

[31] K. Ono, Distribution of the partition function modulo m, Ann. Math. (2) 151 (1) (2000) 293-307.

[32] R.P. Stanley, Enumerative combinatorics. Vol. 1. (English summary) Cambridge Studies in Advanced Mathematics, 49. Cambridge University Press, Cambridge, 1997. 